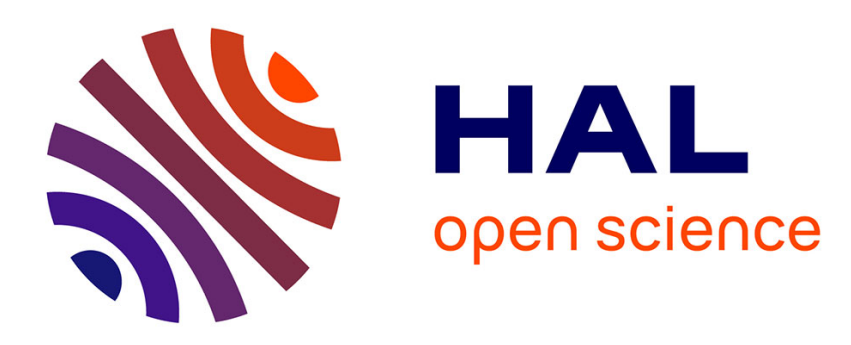

\title{
A fluid model with finite Larmor radius effects for mirror mode dynamics
}

\author{
T. Passot, P.L. Sulem
}

\section{To cite this version:}

T. Passot, P.L. Sulem. A fluid model with finite Larmor radius effects for mirror mode dynamics. Journal of Geophysical Research Space Physics, 2006, 111, pp.04203. 10.1029/2005ja011425 . hal00388635

\section{HAL Id: hal-00388635 \\ https://hal.science/hal-00388635}

Submitted on 4 May 2021

HAL is a multi-disciplinary open access archive for the deposit and dissemination of scientific research documents, whether they are published or not. The documents may come from teaching and research institutions in France or abroad, or from public or private research centers.
L'archive ouverte pluridisciplinaire HAL, est destinée au dépôt et à la diffusion de documents scientifiques de niveau recherche, publiés ou non, émanant des établissements d'enseignement et de recherche français ou étrangers, des laboratoires publics ou privés. 


\title{
A fluid model with finite Larmor radius effects for mirror mode dynamics
}

\author{
T. Passot $^{1}$ and P. L. Sulem ${ }^{1}$ \\ Received 14 September 2005; revised 6 January 2006; accepted 12 January 2006; published 4 April 2006.
}

[1] A fluid model retaining hydrodynamic nonlinearities together with a linear approximation of the Landau damping and of the finite Larmor radius effects is constructed to describe the dynamics of quasi-transverse low-frequency waves in a homogeneous magnetized plasma. It accurately reproduces the kinetic theory predictions for the mirror instability, including its quenching at small transverse scales. The dispersion relation of kinetic Alfvén waves is also recovered. This model should provide an efficient tool for numerical simulations of nonlinear mirror mode dynamics, at least near threshold.

Citation: Passot, T., and P. L. Sulem (2006), A fluid model with finite Larmor radius effects for mirror mode dynamics, J. Geophys. Res., 111, A04203, doi:10.1029/2005JA011425.

\section{Introduction}

[2] The presence of mirror modes has been reported in various space plasma environments, such as the solar wind and the magnetosheath of solar system planets, in regions with a high $\beta$ (ratio of thermal to magnetic pressures) and a strong anisotropy of the proton temperature (dominant in the transverse direction). We refer to Tsurutani et al. [1982], Russel et al. [1999], Huddleston et al. [1999], Treumann et al. [2004] and Pokhotelov et al. [2004] for extended references to observational investigations that were initiated by Kaufman et al. [1970], following the theoretical predictions of Chandrasekhar et al. [1958]. The multispacescraft observations of the Cluster mission have provided an unambiguous detection of mirror modes in the magnetosheath by permitting a distinction between spatial and temporal variations. These extremely low-frequency waves are usually associated with strong depressions of the magnetic field (magnetic holes) that are anti-correlated with the plasma density and propagate very slowly in directions almost perpendicular to the ambient field [see, e.g., Lacombe et al., 1992; Leckband et al., 1995; Schwartz et al., 1996; Lucek et al., 2001; Stasiewicz, 2004]. More rarely, mirror mode structures in the form of magnetic peaks were also observed in regions of very large $\beta$ [see, e.g., Leckband et al., 1995]). It is noticeable that records of the magnetic field intensity by Equator-S satellite revealed peaks, dips and nearly sinusoidal waveforms for the mirror structures, depending on the considered time interval [Lucek et al., 1999].

[3] Recent analysis by Sahraoui et al. [2004, 2006] of turbulent magnetic spectra observed in the magnetosheath indicate that their low-frequency part is dominated by mirror modes with wave vectors quasi-perpendicular to the ambient magnetic field, the magnetic energy appearing to be injected

${ }^{1}$ CNRS, Observatoire de la Côte d'Azur, Nice, France.

Copyright 2006 by the American Geophysical Union. 0148-0227/06/2005JA011425 at a spatial scale associated with the maximum growth rate of the mirror instability. This instability acts as a pumping source for a nonlinear energy cascade extending to scales at least as small as a tenth of the proton gyroradius.

[4] As discussed in detail by Gary [1992] and McKean et al. [1994], depending on the plasma parameters, the mirror instability can be competing with the ion cyclotron anisotropy instability, but the former clearly dominates for large $\beta$ (typically larger than 5). We concentrate here on the mirror instability whose relevance can also be enhanced by the presence of helium $\mathrm{He}^{++}$[McKean et al., 1994]. The ion cyclotron anisotropy instability, being purely kinetic, is not amenable to a fluid description.

[5] As noted by Treumann et al. [2004], there exists so far no reliable nonlinear theory for the formation and evolution of the mirror modes in high temperature plasmas. An interesting phenomenological description of the mirror instability is given by Southwood and Kivelson [1993] and comparisons with hybrid simulations are presented by McKean et al. [1992, 1993]. A fluid closure suggested by magnetosheath observations and by these simulations was proposed by Gary et al. [1994]. Simple saturated solutions, based on the separation of the particle distribution into trapped and untrapped components that respond differently to the magnetic field variations, are discussed by Kivelson and Southwood [1996], Pantellini et al. [1995] and Pantellini [1998]. It is of interest to note that the model presented in the last reference reproduces the different forms of mirror structures, dips or humps, depending on the moderate or large (typically larger than 10) value of the plasma $\beta$. An analogy between mirror instability and superconductivity is presented by Treumann et al. [2004], who predict a scaling law for the variation of the critical magnetic field with the temperature anisotropy. We also mention that Stasiewicz [2004, 2005] interprets the magnetosheath structures usually considered as mirror modes, as trains of slow-mode magnetosonic solitons defined as exact solutions of Hall-MHD equations with anisotropic pressure and negligible gyroviscosity. The relevance of such a 
description for the terrestrial magnetosheath can however be questioned, due to the small-scale character of the structures and the high values of the plasma $\beta$ that strengthen the importance of kinetic effects. Velocity fluctuations are actually to be considered to discriminate between mirror and slow magnetosonic waves [Lin et al., 1998].

[6] The aim of the present paper is to construct a fluid model retaining the main kinetic effects, suitable to address the formation and evolution of magnetic structures resulting from the nonlinear development of the mirror instability. Although nonlinear kinetic effects may contribute to the saturation of the mirror instability, it is of interest to first focus on the role of hydrodynamic nonlinearities that can be dominant near the instability threshold and that may be at the origin of the observed power law spectra. Nevertheless, a specific property of the mirror modes that makes their description difficult using fluid models, originates from the increase of the maximal instability growth rate with the transverse wave number up to a scale comparable with the ion Larmor radius. As a consequence, in spite of the accurate estimates of the large-scale instability growth rate obtained by Snyder et al. [1997] and Bugnon et al. [2006] using fluid models with Landau damping (Landau fluids), numerical integrations in the nonlinear regime are hardly feasible in the presence of the mirror instability because the smallest scales retained by the spatial discretization turn out to be the most unstable. In this context, it is interesting to mention the approach of Baumgärtel [2001], who used onedimensional MHD equations with a gyrotropic pressure tensor where the parallel and transverse pressures obey a double polytropic closure in terms of the local density and magnetic field, with exponents departing by $20 \%$ from those associated with isothermality. The mirror instability is suppressed at small length scales by applying a filtering procedure in Fourier space. It is noticeable that this model is sufficient to reproduce the formation of magnetic dips anticorrelated with density humps, typical of mirror mode structures (but also of slow modes).

[7] Improved Landau fluid models including small-scale finite Larmor radius (FLR) corrections are necessary to accurately capture the quenching of the instability at these scales. The main goal of the present paper is to develop such a model, where nongyrotropic contributions are evaluated in the framework of the linear kinetic theory and included in a convenient way within a Landau fluid description. Approachs involving nonlinear fluid equations, together with the evaluation of suitable quantities using the linear kinetic theory, were also used by Smolyakov et al. [1995] and Cheng and Johnson [1999] in other contexts. The present model, that allows for an accurate description of quasitransverse dynamics, appears to be as simple as possible in the sense that the fluid hierarchy is closed at the level of the pressure tensor. Even though it allows for adiabatic behavior, it is best suited for the simulation of quasi-isothermal dynamics. We should here mention that for anisotropic plasmas, the so-called isothermal limit obtained when the ratio of typical phase velocities to thermal velocities along the ambient field tends to zero, corresponds in the linear case to a truly isothermal behavior of the parallel temperature but prescribes a variation of the perpendicular temperature with magnetic field fluctuations. A nonlinear dependency of the perpendicular temperatures in terms of the local magnetic field can in fact be retained, as discussed in section 4.3 , but the parallel temperatures remain unaffected.

[8] In order to validate the model, comparisons with the full kinetic theory are performed at the level of the kinetic Alfvén wave (KAW) dispersion relation and of the linear mirror instability. This instability was investigated by Gary [1992] using a direct numerical approach in a broad range of regimes and in a series of papers by Pokhotelov and coworkers, using asymptotic expansions based on the ultra low frequency of the mirror modes compared with the ion gyrofrequency $\Omega$. We nevertheless revisit it here, with two main goals: (i) we evaluate the accuracy of various approximations usually made by the analytical approaches, concerning the plasma response function and the truncation of the kinetic formulas at dominant order in the direction parameter $k_{z} / k_{\perp}$; (ii) we investigate the effect of warm electrons, a regime that was analytically considered by Pokhotelov et al. [2000] in the context of a large-scale analysis only. Detailed comparisons are in particular performed with Pokhotelov et al. [2004], who studied the stability of modes with wavelengths extending to and beyond the ion Larmor radius in the case of cold electrons. Performing these comparisons led us to first consider values of proton $\beta$ close to unity, although at such values of $\beta$, the anisotropic ion cyclotron (AIC) instability is usually dominant. Comparisons between the kinetic theory and the fluid model for $\beta$ large enough for the mirror instability to be dominant, are also presented. While the linear stability analysis is usually performed by introducing the dielectric tensor, it is more convenient for our purpose to deal directly with Maxwell equations supplemented by the kinetic expressions for the densities and velocities of the ions and electrons. The two approaches are obviously equivalent but the expressions of these hydrodynamic quantities as given by the kinetic theory are important ingredients to build the fluid closure. Because of the very low frequency $\omega$ of the mirror modes, the analysis is performed in the framework of a perturbative expansion of the particle distribution functions in terms of the small parameter $\omega / \Omega$. All the contributions arising beyond the leading order have in fact an unequal influence on the instability growth rate, which leads us to select those terms that are, in practice, to be retained in a Landau fluid whose formulation is aimed to be as simple as possible.

[9] In addition to the computation of the mirror instability growth rate for perturbations with arbitrary transverse scale, the present model accurately reproduces the KAW dispersion relation, but only the phase velocity of oblique magnetosonic waves. Capturing the full dispersion relation of magnetosonic and Alfvén waves whose propagation direction makes an intermediate angle with the magnetic field would require a higher order closure and, as a consequence, a more refined matching of the fluid and kinetic theories.

[10] The outline of the paper is as follow. In section 2, we use the linearized Vlasov-Maxwell system to obtain, in a low-frequency asymptotic, the kinetic expressions of the number density of the particles together with the velocity of each species. Section 3 presents an analysis of the dispersion relation for modes propagating in a direction quasiperpendicular to the ambient field and includes comparisons with previous works. A main goal is to evaluate the accuracy of the usually performed approximations and to 
select among all the terms arising at the same order in a lowfrequency expansion of the hydrodynamic moments, the contributions that have a strong effect on the mirror instability growth rate and on the KAW dispersion relation. The contributions with a subdominant influence will be neglected when designing closure formulas. Section 4 , that can be viewed as the main section of this paper, is devoted to the derivation of the fluid model together with its validation by comparison with the results of section 3 . Section 5 is a brief conclusion that summarizes the capability and limits of the present model and suggests further developments.

\section{Low-Frequency Linear Kinetic Theory}

[11] Consider a spatially homogeneous uniformly magnetized plasma with a bi-Maxwellian distribution function at equilibrium

$$
f_{r}^{(0)}=\frac{1}{(2 \pi)^{3 / 2}} \frac{m_{r}^{3 / 2}}{T_{\perp r}^{(0)} T_{\| r}^{(0) 1 / 2}} \exp \left\{-\left(\frac{m_{r}}{2 T_{\| r}^{(0)}} v_{\|}^{2}+\frac{m_{r}}{2 T_{\perp r}^{(0)}} v_{\perp}^{2}\right)\right\}
$$

for the particles of species $r$ with charge $q_{r}$, mass $m_{r}$, and average number density $n_{r}$, in the absence of net charges or currents. The temperatures are measured in energy units (being implicitly multiplied by the Bolzmann constant). In addition to its convenience for analytical calculations, this distribution provides a reasonable description of the plasma as it flows from the Earth's quasi-perpendicular bow shock deeper into the magnetosphere [Sckopke et al., 1990; McKean et al., 1995]. The Vlasov equation for each species is linearized about $f_{r}^{(0)}$, writing $f_{r}=f_{r}^{(0)}+f_{r}^{(1)}$, with $B=B_{0} \widehat{z}+$ $B^{(1)}$ and $E=E^{(1)}, B_{0}$ denoting the amplitude of the ambient magnetic field taken in the z-direction (no background electric field). In the following we denote by $b_{x}, b_{y}$ and $b_{z}$ the components of $B^{(1)}$. The Vlasov equation is supplemented by Maxwell equations that express the electric and magnetic fields $E$ and $B$ in terms of the current $j=\sum_{r} q_{r} n_{r}$ $\int v f_{r} d^{3} v$ and the total charge $v=\sum_{r} q_{r} n_{r} \int f_{r} d^{3} v$, in the usual form $\partial_{t} B=-c \nabla \times E, c \nabla \times B=4 \pi j+\partial_{t} E$ and $\nabla \cdot E=$ $4 \pi \nu$.

[12] It is usual to introduce the scalar potentials $\Phi$ and $\Psi$ together with the vector potential $A$ in the form $E_{\perp}=$ $-\nabla_{\perp} \Phi-(1 / c) \partial_{t} A_{\perp}, E_{z}=-\partial_{z} \Psi$ and $B=B_{0}+\nabla \times A$ with $\nabla \cdot A=0$. It follows that $A_{z}=\left(c k_{z} / \omega\right)(\Phi-\Psi)$, where, since there is no ambiguity, we use the same notation for a field and its Fourier transform.

[13] Assuming a plasma made of protons (subscript $\mathrm{p}$ ) and electrons (subscript e) with charge $q_{p}=-q_{e}=e$, one rewrites the Ampère-Maxwell equation in the nondimensional form

$$
\frac{c_{A}^{2}}{c^{2}} b\left(1+\frac{k_{z}^{2}}{k_{\perp}^{2}}\right) \frac{e \Phi}{T_{\perp p}^{(0)}}=\frac{n_{p}}{n^{(0)}}-\frac{n_{e}}{n^{(0)}}
$$

Here we introduce the standard notation (to be distinguished from the magnetic field components that involve subscripts) $b=T_{\perp p}^{(0)} k_{\perp}^{2} /\left(m_{p} \Omega^{2}\right)$ as a measure of the square transverse wavenumber, where $\Omega=e B_{0} /\left(m_{p} c\right)$ denotes the proton gyrofrequency, and $c_{A}=B_{0} / \sqrt{4 \pi m_{p} n^{(0)}}$ is the Alfvén speed.
Assuming $c_{A} \ll c$ implies local electric neutrality $n_{p}=n_{e}$. Under this assumption, one can also neglect the displacement current and get

$$
k^{2}(\Phi-\Psi)=-\frac{4 \pi}{c^{2}} \frac{\omega}{k_{z}} j_{z} .
$$

One also has $(\widehat{z}$ being the unit vector along the ambient field and arrows being used to discriminate between transverse vector components from their moduli)

$$
k^{2} b_{z}=i \frac{4 \pi}{c}\left(\vec{k}_{\perp} \times \vec{j}_{\perp}\right) \cdot \widehat{z},
$$

where the ion and electron densities and velocities needed to express the current $j$ (of parallel and transverse components $j_{z}$ and $\vec{j}_{\perp}$ ) are to be computed using the kinetic theory.The transverse magnetic field components are given by

$$
\begin{aligned}
& b_{x}=-\frac{k_{x} k_{z}}{k_{\perp}^{2}} b_{z}+i \frac{c}{\omega} k_{y} k_{z}\left(1+\frac{k_{z}^{2}}{k_{\perp}^{2}}\right)(\Phi-\Psi) \\
& b_{y}=-\frac{k_{y} k_{z}}{k_{\perp}^{2}} b_{z}-i \frac{c}{\omega} k_{x} k_{z}\left(1+\frac{k_{z}^{2}}{k_{\perp}^{2}}\right)(\Phi-\Psi) .
\end{aligned}
$$

[14] It is convenient to express the velocity $v$ in a cylindrical coordinate system by defining the azimuthal angle $\phi=$ $\tan ^{-1}\left(v_{y} / v_{x}\right)$ and writing $v=\left(v_{\perp} \cos \phi, v_{\perp} \sin \phi, v_{\|}\right)$and $\nabla_{v}=$ $\left(\cos \phi \partial_{v_{\perp}}-\left(\sin \phi / v_{\perp}\right) \partial_{\phi}, \partial_{v_{\perp}}+\left(\cos \phi / v_{\perp}\right) \partial_{\phi}, \partial_{v_{\|}}\right)$. Restricting ourselves to the case of linear perturbations in the form of plane waves of wavevector $k=\left(k_{x}=k_{\perp} \cos \psi\right.$, $\left.k_{y}=k_{\perp} \sin \psi, k_{z}\right)$, such that $E^{(1)}=\widetilde{E}^{(1)} \exp (i(k \cdot x-\omega t))+$ c.c., $B^{(1)}=\widetilde{b}^{(1)} \exp (i(k \cdot x-\omega t))+$ c.c. and $f_{r}^{(1)}=\widetilde{f}_{r}^{(1)} \exp (i(k$. $x-\omega t))+$ c.c., and using Faraday-Maxwell equation to write $\widetilde{B}^{(1)}=(c / \omega)\left(k \times \widetilde{E}^{(1)}\right)$, one has (dropping the tildes)

$$
\begin{aligned}
& -i(\omega-k \cdot v) f_{r}^{(1)}-\frac{q_{r} B_{0}}{m_{r} c} \frac{\partial f_{r}^{(1)}}{\partial \phi} \\
& =-\frac{q_{r}}{m_{r}}\left[\left(E^{(1)}\left(1-\frac{k \cdot v}{\omega}\right)+\frac{k\left(v \cdot E^{(1)}\right)}{\omega}\right) \cdot \nabla_{v} f_{r}^{(1)}\right] .
\end{aligned}
$$

For a bi-Maxwellian distribution, following Akhiezer et al. [1975], we get for protons

$$
\begin{aligned}
f_{p}^{(1)}= & -e f_{p}^{(0)} \sum_{l, n=-\infty}^{+\infty} \frac{J_{n}\left(\lambda_{p}\right) \exp (i(n-l)(\phi-\psi))}{l \Omega+k_{z} v_{\|}-\omega} \\
& \cdot\left\{\left[\frac{1}{T_{\perp p}^{(0)}}+\frac{k_{z} v_{\|}}{\omega}\left(\frac{1}{T_{\| p}^{(0)}}-\frac{1}{T_{\perp p}^{(0)}}\right)\right]\right. \\
& \times\left[l \Omega\left(1+\frac{k_{z}^{2}}{k_{\perp}^{2}}\right) J_{l}\left(\lambda_{p}\right) \Phi-\frac{k_{z}^{2}}{k_{\perp}^{2}} l \Omega J_{l}\left(\lambda_{p}\right) \Psi-\frac{\omega v_{\perp}}{k_{\perp} c} J_{l}^{\prime}\left(\lambda_{p}\right) b_{z}\right] \\
& \left.+\left[\frac{k_{z} v_{\|}}{T_{\| p}^{(0)}}-\frac{l \Omega k_{z} v_{\|}}{\omega}\left(\frac{1}{T_{\| p}^{(0)}}-\frac{1}{T_{\perp p}^{(0)}}\right)\right] J_{l}\left(\lambda_{p}\right) \Psi\right\}
\end{aligned}
$$

where $\lambda_{p}=k_{\perp} v_{\perp} / \Omega$ and $J_{l}$ is the Bessel function of order $l$. A similar equation is obtained for the electrons, by replacing $m_{p}$ by $m_{e}$ and $e$ by $-e$ everywhere, including in the gyrofrequency.

[15] The fluid moments are computed in Appendix A, as an expansion to order $\omega^{2} / \Omega^{2}$, keeping the ratio $\omega / k_{z}$ a priori finite. In the resulting expressions, we separate the main contributions that are to be retained, from additional terms $\mathcal{S}_{n}(n=1,2,3)$ expressed in Appendix B, that will be shown 
to have a negligible effect on the mirror instability growth rate and the KAW dispersion relation.

[16] For the perturbations of the number density of the protons, we get

$$
\begin{aligned}
\frac{n_{p}^{(1)}}{n_{p}^{(0)}}= & \left(\Gamma_{1}(b)-\Gamma_{0}(b)\right)\left(\frac{T_{\perp p}^{(0)}}{T_{\| p}^{(0)}} R\left(\zeta_{p}\right)-1\right) \frac{b_{z}}{B_{0}}-\Gamma_{0}(b) R\left(\zeta_{p}\right) \frac{e \Psi}{T_{\| p}^{(0)}} \\
& -\frac{e}{T_{\perp p}^{(0)}}\left(1-\Gamma_{0}(b)\right)\left(\Phi+\frac{k_{z}^{2}}{k_{\perp}^{2}}(\Phi-\Psi)\right)+\mathcal{S}_{1} .
\end{aligned}
$$

[17] The plasma response function entering the above equation is defined as $R(\zeta)=1+\zeta Z(\zeta)$ where $Z(\zeta)$ is the plasma dispersion function. Furthermore, $\Gamma_{\nu}(b)=e^{-b} I_{\nu}(b)$ where $I_{\nu}(b)$ is the modified Bessel function of order $\nu$.

[18] For the electrons, when neglecting contributions of order $m_{e} / m_{p}$, one simply has

$$
\frac{n_{e}^{(1)}}{n_{e}^{(0)}}=-\left(\frac{T_{\perp e}^{(0)}}{T_{\| e}^{(0)}} R\left(\zeta_{e}\right)-1\right) \frac{b_{z}}{B_{0}}+R\left(\zeta_{e}\right) \frac{e \Psi}{T_{\| e}^{(0)}} .
$$

The ion parallel velocity is given by

$u_{z p}=\frac{T_{\perp p}^{(0)}}{T_{\| p}^{(0)}} \frac{\omega}{k_{z}} R\left(\zeta_{p}\right)\left[\left(\Gamma_{1}(b)-\Gamma_{0}(b)\right) \frac{b_{z}}{B_{0}}-\Gamma_{0}(b) R\left(\zeta_{p}\right) \frac{e \Psi}{T_{\perp p}^{(0)}}\right]+\mathcal{S}_{2}$.

For the electrons, one has

$$
\begin{aligned}
u_{z e}= & -\frac{T_{\perp e}^{(0)}}{T_{\| e}^{(0)}} \frac{\omega}{k_{z}} R\left(\zeta_{e}\right)\left[\frac{b_{z}}{B_{0}}-R\left(\zeta_{e}\right) \frac{e \Psi}{T_{\perp e}^{(0)}}\right] \\
& +\frac{k_{z}}{\omega} \frac{T_{\perp e}^{(0)}-T_{\| e}^{(0)}}{m_{p}} b \frac{e}{T_{\perp p}^{(0)}}\left(1+\frac{k_{z}^{2}}{k_{\perp}^{2}}\right)(\Phi-\Psi) .
\end{aligned}
$$

[19] The transverse hydrodynamic velocity of each particle species $r$ is conveniently decomposed into compressible and solenoidal parts by writing

$$
u_{\perp r}=-\nabla_{\perp} \chi_{c r}+\nabla_{\perp} \times\left(\chi_{s r} \widehat{z}\right) .
$$

One has for the ions

$$
\begin{aligned}
\chi_{s p}= & \frac{1}{\Omega} \frac{T_{\perp p}^{(0)}}{m_{p}}\left(\Gamma_{0}(b)-\Gamma_{1}(b)\right)\left[2\left(\frac{T_{\perp p}^{(0)}}{T_{\| p}^{(0)}} R\left(\zeta_{p}\right)-1\right) \frac{b_{z}}{B_{0}}\right. \\
& \left.+R\left(\zeta_{p}\right) \frac{e \Psi}{T_{\| p}^{(0)}}-\frac{e}{T_{\perp p}^{(0)}}\left(\Phi+\frac{k_{z}^{2}}{k_{\perp}^{2}}(\Phi-\Psi)\right)\right] \\
& -4 \frac{T_{\perp p}^{(0)}-T_{\| p}^{(0)}}{m_{p}} \frac{k_{z}^{2}}{k_{\perp}^{2} \Omega} C_{1}^{3}(b) \frac{b_{z}}{B_{0}}+\mathcal{S}_{3},
\end{aligned}
$$

where $C_{1}^{3}(b)$ is defined in Appendix A. Furthermore,

$$
\begin{aligned}
\chi_{c p}= & \frac{i \omega}{k_{\perp}^{2}}\left(\Gamma_{0}(b)-\Gamma_{1}(b)\right) \frac{b_{z}}{B_{0}}+\left(1-\Gamma_{0}(b)\right) \frac{i}{\omega} \frac{k_{z}^{2}}{k_{\perp}^{2}} \\
& \times \frac{e}{T_{\perp p}^{(0)}}\left[\frac{T_{\perp p}^{(0)}-T_{\| p}^{(0)}}{m_{p}}\left(1+\frac{k_{z}^{2}}{k_{\perp}^{2}}\right)(\Phi-\Psi) .\right. \\
& \left.-\frac{\omega^{2}}{k_{z}^{2}}\left(\Phi+\frac{k_{z}^{2}}{k_{\perp}^{2}}(\Phi-\Psi)\right)\right] .
\end{aligned}
$$

Similarly, one has for the electrons

$$
\begin{aligned}
\chi_{s e}= & -\frac{1}{\Omega} \frac{T_{\perp e}^{(0)}}{m_{p}}\left[2\left(\frac{T_{\perp e}^{(0)}}{T_{\| e}^{(0)}} R\left(\zeta_{e}\right)-1\right) \frac{b_{z}}{B_{0}}\right. \\
& \left.-R\left(\zeta_{e}\right) \frac{e \Psi}{T_{\| e}^{(0)}}+\frac{e}{T_{\perp e}^{(0)}}\left(\Phi+\frac{k_{z}^{2}}{k_{\perp}^{2}}(\Phi-\Psi)\right)\right] \\
& -\frac{T_{\perp e}^{(0)}-T_{\| e}^{(0)}}{m_{p}} \frac{k_{z}^{2}}{k_{\perp}^{2} \Omega} \frac{b_{z}}{B_{0}}
\end{aligned}
$$

and

$$
\chi_{c e}=\frac{i \omega}{k_{\perp}^{2}} \frac{b_{z}}{B_{0}}
$$

[20] Note that in the second order contributions arising in the above expressions, we separated the term proportional to $C_{1}^{3}(b)$ in equation (13) that keeps a finite limit $\mathcal{C}_{1}^{3} \rightarrow 1 / 4$ as $b \rightarrow 0$, from the terms denoted $\mathcal{S}_{n}(n=1,2,3)$ that involve coefficients that tend to zero in this limit. The latter coefficients nevertheless reach significant values when $b$ is taken finite. Their effect on the mirror instability growth rate in this range of scales thus requires a more detailed study, performed in the next section.

\section{Linear Dynamics of Quasi-Perpendicular Modes}

[21] Substituting the expressions for the density and for the parallel and perpendicular velocities provided by the kinetic theory into equations (1)-(3) leads to a system of homogeneous linear equations easily solved using a symbolic calculator. Possible simplifications such as neglecting $\mathcal{S}_{n}(n=1,2,3)$ contributions announced in the previous section are however to be validated. Furthermore, the plasma response function is conveniently replaced by a Padé approximant, an operation whose accuracy is to be evaluated. For this purpose, the influence of these approximations on the dispersion relations of different waves in various regimes are considered.

\subsection{Dispersion Relation of Kinetic Alfvén Waves}

[22] For kinetic Alfvén waves in the regime $b \ll 1$ and $\theta=k_{z} / k_{\perp} \ll 1$, with isotropic equilibrium temperatures such that $\tau \equiv T_{\| e}^{(0)} / T_{\| p}^{(0)} \gg 1$, the dispersion relation was asymptotically derived by Hasegawa and Chen [1975, 1976] in the form $\omega^{2}=k_{z}^{2} v_{A}^{2}\left(1+\left(\frac{3}{4}+\frac{T_{e}^{(0)}}{T_{p}^{(0)}}\right) b\right)$. The resolution of our linear system was performed using a fourth pole approximation of the plasma response function $R$, the $\mathcal{S}_{n}$ terms being neglected. Best agreement with this asymptotic description is found when $\beta_{\perp p} \equiv 8 \pi p_{\perp p}^{(0)} / B_{0}^{2} \ll$ 1 and $\tau \gg 1$, excluding however extreme values. An example is shown in Figure 1 that displays $\Re(\omega) / k_{z} v_{A}$ as a function of $b$ for $\theta=10^{-3}, \beta_{\perp p}=0.001, \tau=100$ and $A_{p}=A_{e}=$ 0 (where the anisotropy factor is defined by $A_{r}=T_{\perp r}^{(0)} / T_{\| r}^{(0)}-1$ ), for both the numerical resolution of the dispersion relation (circles) and the above analytic formula (diamonds). The agreement is excellent at the largest scales where the formula is asymptotically exact, while the discrepancy approaches $20 \%$ for $b=0.1$. The $\mathcal{S}_{n}$ terms are checked 


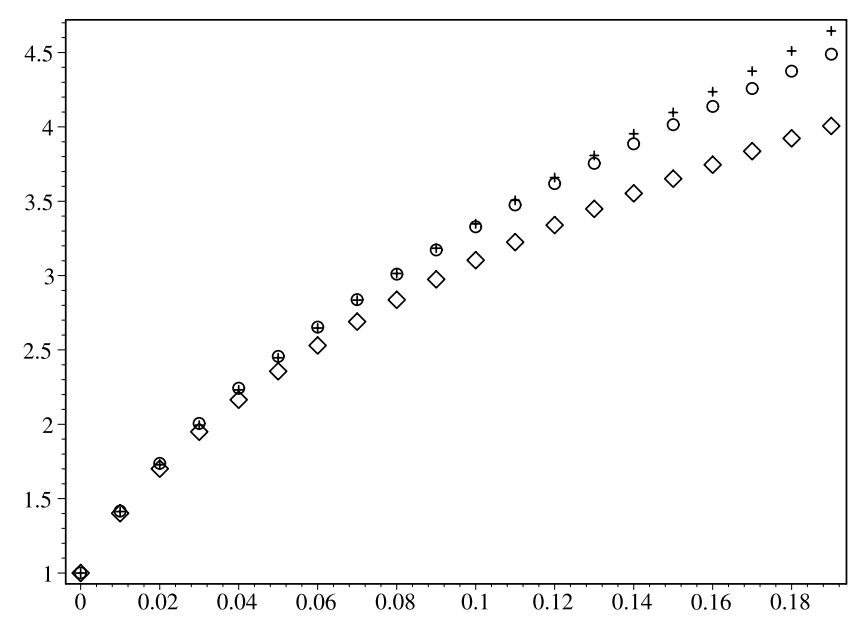

Figure 1. Comparison of the normalized frequencies $\Re(\omega) / k_{z} v_{A}$ of KAWs as a function of $b$ for $\theta=10^{-3}, \beta_{\perp p}=$ $0.001, \tau=100$ and isotropic equilibrium temperatures, obtained from the full dispersion relation (circles) and from the analytic formula $\omega^{2}=k_{z}^{2} v_{A}^{2}\left(1+\left(\frac{3}{4}+\frac{T_{e}^{(0)}}{T_{p}^{(0)}}\right) b\right)$ (diamonds). The crosses refer to the predictions of the model described in section 4.

to have a totally negligible effect for the above value of $\theta$. For $\theta=10^{-1}$ their effect is still negligible at very large scales but leads to a $3 \%$ difference at $k \cdot r_{p}=0.2$, which is still satisfactory.

\subsection{Mirror Mode Instability}

[23] Using the dispersion relation obtained with equations (1)-(3), we display in Figure 2 the mirror mode growth rate $\Im(\omega) /\left(k_{\perp} v_{t h, p}\right)$ as a function of the direction parameter $\theta=k_{z} / k_{\perp}$ for the case where $A_{p}=1, A_{e}=0.2, \tau=$ 1 and $\beta_{\perp p}=2$, using the full plasma response function $R$ (circles) and its one-pole approximation (crosses), together

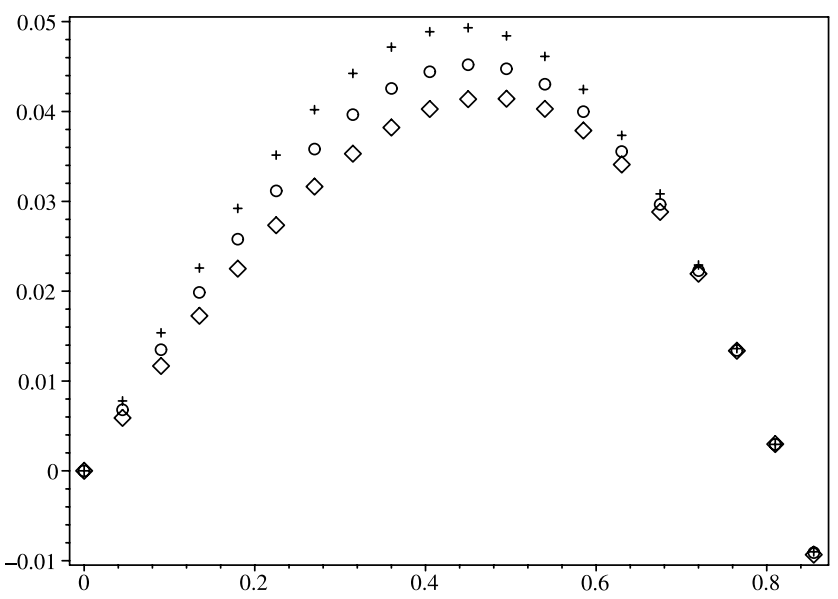

Figure 2. Mirror mode growth rate $\Im(\omega) / k_{\perp} v_{t h, p}$ as a function of $\theta=k_{z} / k_{\perp}$ for a case with $A_{p}=1, A_{e}=0.2, \tau=1$, $\beta_{\perp p}=2$ using the full plasma response function $R$ (circles) and its one-pole approximation (crosses), together with the growth rate given by formula (23) from Pokhotelov et al. [2000] (diamonds), based on the "quasi-hydrodynamic approximation." with the growth rate given by formula (23) from Pokhotelov et al. [2000] that is based on the "quasi-hydrodynamic approximation" for the large-scale dynamics (diamonds). A significant deviation takes place between the three curves when the growth rate increases, indicating a strong sensitivity to the level of approximation of the plasma response function. In contrast, neglecting the $\mathcal{S}_{n}$ terms but keeping the full function $R$, leads to a growth rate whose values all fall within the circle symbols corresponding to the full calculation.

[24] We now address the behavior of the instability growth rate as a function of the parameter $b$ that measures the square ratio of the transverse scale of the perturbation to the proton Larmor radius $r_{p}$. The main result of the numerical calculation of Gary [1992] (see his Figure 2 for electrons with a nonzero isotropic temperature) and of the asymptotic analysis by Pokhotelov et al. [2004] in the case of cold electrons concerns the increase of the instability threshold at small wavelength and its disappearance for scales smaller than a fraction of the Larmor radius. It is of interest to reconsider this latter case and in particular to investigate here as well the role of the terms proportional to $\theta^{2}$. Figure 3 displays $\gamma_{\max } / \Omega$, where $\gamma_{\max }$ is the growth rate maximized over the angle of propagation, as a function of $k_{\perp} r_{p}=\sqrt{2 b}$ for $A_{p}=\beta_{\perp p}=1.5$ and $T_{e}=0$, that are the parameters of Figure 1 of Pokhotelov et al. [2004]. Diamonds and circles correspond to the growth rate calculated from the kinetic dispersion relation with and without the $\mathcal{S}_{n}$ terms. It is clear from this picture that these terms can be omitted whatever the value of $\theta$ and $b$. Crosses correspond to the growth rate calculated using a series expansion of the dispersion relation truncated at order $\zeta^{3}$. These values are closer to those given by Pokhotelov et al. [2004] using a low-order approximation of the function $R$. For this case again, it appears that the results are most sensitive to the degree of approximation of the plasma dispersion function. Another remark concerns the value of $\theta$ at which the growth rate is maximum. We find for $\left(k_{z} / k_{\perp}\right)_{\max }$ values of the order of a few tenths that are thus, up to the precision of our

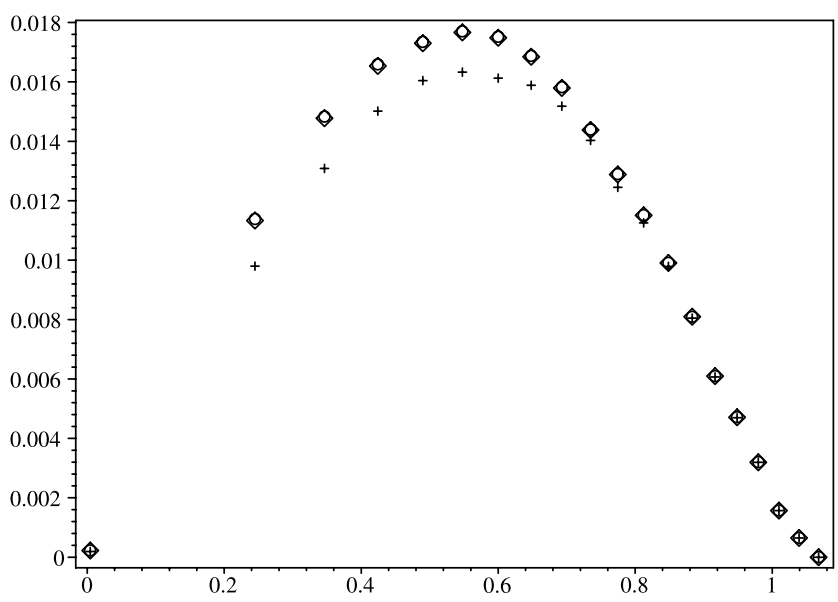

Figure 3. Growth rates $\gamma_{\max } / \Omega$ maximized over the angle of propagation, as a function of $k_{\perp} r_{p}=\sqrt{2 b}$ for $A_{p}=\beta_{\perp p}=$ 1.5 and cold electrons, using the kinetic dispersion relation calculated with (diamonds) and without (circles) $\mathcal{S}_{n}$ terms. Crosses correspond to the growth rate calculated using a series expansion of the dispersion relation truncated at order $\zeta^{3}$ (see text). 


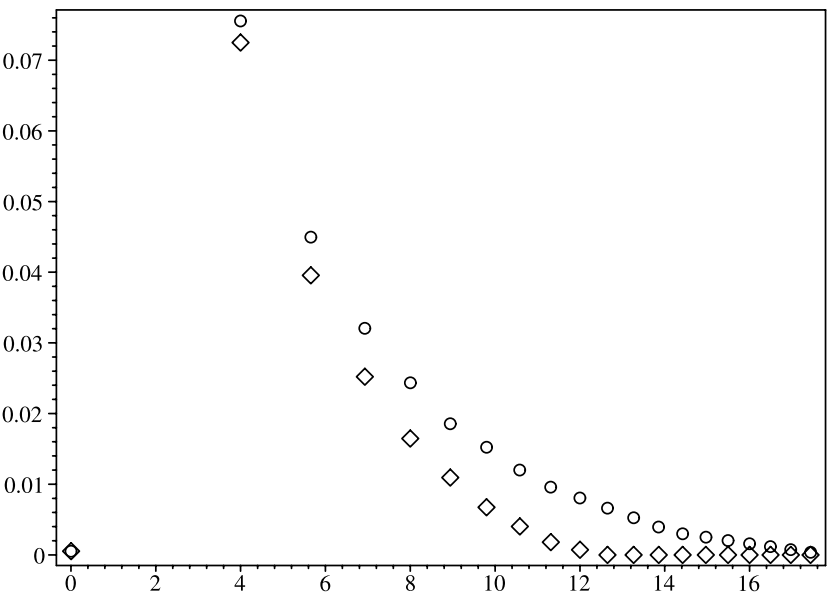

Figure 4. Growth rate (maximized over the propagation angles) as a function of $k_{\perp} r_{p}=\sqrt{2 b}$, for electron temperature anisotropies $A_{e}=1$ (diamonds) and $A_{e}=$ 1.005 (circles), when $A_{p}=1.5, \tau=1$ and $\beta_{\perp p}=1.5$.

graph (not shown), exactly 10 times larger than those displayed in Figure 3 of Pokhotelov et al. [2004] where a typo is suspected. The magnitude of the angle associated with the maximum growth rate is of importance since the expansion used in the calculation of the perturbed distribution function is based on the assumption of small $\theta$. The above remark on the irrelevance of the terms proportional to $\theta^{2}$ nevertheless ensures the validity of the present calculations.

[25] The case of warm electrons is more delicate. As mentioned by Gary [1992], Pantellini and Schwartz [1995] and Pokhotelov et al. [2000], in the limit $b=0$ the instability growth rate is very sensitive to the electron temperature anisotropy. When the latter is strong, the behavior of this instability at small wavelength can in fact hardly be estimated using the present formalism, as the growth rate rapidly reaches values which fall outside the range of the small frequency approximation. We display in Figure 4 the growth rate (maximized over the propagation angles) for two electron temperature anisotropies, namely $A_{e}=1$ (diamonds) and $A_{e}=1.005$ (circles), when $A_{p}=1.5, \tau=$ 1 and $\beta_{\perp p}=1.5$. As seen on this graph, the value of $k_{\perp} r_{p}$ at which the instability disappears is increased by more than an order of magnitude compared to the case $A_{e}=0$. These observations on the effect of the electron temperature anisotropy are consistent with the results of Génot et al. [2001]. At this distance from threshold, this magnitude of electron temperature anisotropy appears to be at the limit of validity of the present ordering.

\section{A Fluid Model for Mirror Modes}

[26] The idea is to build a Landau fluid model by supplementing the usual MHD equations not only with a description of the linear Landau damping but also with information about the small transverse scales, as provided by the linear kinetic theory, with the aim to arrest the mirror instability at small scale. Different models can a priori be constructed with various levels of complexity. In this paper, we restrict ourselves to the simple framework where the fluid hierarchy is closed at the level of the pressure tensor of each particle species.

\subsection{Fluid Hierarchy}

[27] Using electric neutrality, one defines as usual the proton density $\rho_{p}=m_{p} n$, and neglects terms proportional to $m_{e} / m_{p}$. The proton and electron velocities are related by $u_{e}=$ $u_{p}-j /(e n)$. The ion pressure tensor is rewritten as the sum $\mathbf{p}_{p}=p_{\perp p}(\mathbf{I}-\widehat{b} \otimes \widehat{b})+p_{\| p} \widehat{b} \otimes \widehat{b}+\boldsymbol{\Pi}$ (where $\widehat{b}$ is the unit vector along the local magnetic field) of the gyrotropic and gyroviscous contributions, while the electron pressure tensor is taken gyrotropic and characterized by the parallel and transverse pressures $p_{\| e}$ and $p_{\perp e}$. One has the usual equations

$$
\begin{gathered}
\partial_{t} \rho_{p}+\nabla \cdot\left(\rho_{p} u_{p}\right)=0 \\
\partial_{t} u_{p}+u_{p} \cdot \nabla u_{p}+\frac{1}{\rho_{p}} \nabla \cdot \mathbf{p}_{p} \\
-\frac{e}{m_{p}}\left(E+\frac{1}{c} u_{p} \times B\right)=0 \\
E=-\frac{1}{c}\left(u_{p}-\frac{j}{n e}\right) \times B-\frac{1}{n e} \nabla \cdot \mathbf{p}_{e}
\end{gathered}
$$

together with the Faraday-Maxwell equation for the magnetic field.

[28] The above hierarchy is to be closed by prescribing the pressure tensors. At the level of the linear kinetic theory, all their components, are given in terms of $b_{z}, \Phi$ and $\Psi$ (see section 4.2). Nevertheless, since these expressions involve the plasma response function (and thus nonlocal operators in the time variable), they cannot be conveniently substituted into the fluid equations, even in a model where the pressure fluctuations are retained at a linear level only.

[29] Note that equation (19) neglects electron inertia. This point was questioned by Pokhotelov et al. [2000], especially when the electrons are hot. Their statement is based on the fact that when substituting the kinetic expression for the pressure within the equation for the electron longitudinal velocity in order to get the potential $\Psi$, a cancellation takes place, which makes the acceleration term relevant in this equation in spite of its smallness. The resulting expression for $\Psi$ provided by equation (21) of Pokotelov et al. [2000] can in fact be reproduced by substituting the kinetic expression of $j$ within equation (2) (where the 1.h.s. turns out to be negligible at large scale). This suggests that equation (19) can be kept as it is when used in a fluid description. In fact, comparison with the kinetic theory shows that the resulting error is subdominant compared to that resulting from a low order approximation of the plasma response function.

\subsection{Kinetic Expressions of the Pressure Tensors}

[30] We hereafter show that one can express the linearized temperature fluctuations (directly related to the gyrotropic pressure disturbances) together with the gyroviscosity stress tensor in a form suitable to be implemented in the fluid formalism. The analysis starts with the kinetic description of the pressure tensor. In the linear approximation, the elements of the pressure tensor perturbations reduce to $p_{i j}^{(1)}=n^{(0)} m \int$ $v_{i} v_{j} f^{(1)} d^{3} v$. Furthermore, $\widehat{b}_{x}=b_{x} / B_{0}, \widehat{b}_{y}=b_{y} / B_{0}$ and $\widehat{b}_{z}=1$. One has $p_{\perp}^{(1)}=n^{(0)} m \int\left(v_{\perp}^{2} / 2\right) f^{(1)} d^{3} v$ and $p_{\|}^{(1)}=n^{(0)} m \int$ $v_{\|}^{2} f^{(1)} d^{3} v$, from which one easily derives the parallel and transverse temperature perturbations $T_{\|}^{(1)} / T_{\|}^{(0)}=p_{\|}^{(1)} / p_{\|}^{(0)}-$ $\rho^{(1)} / \rho^{(0)}$ and $T_{\perp}^{(1)} / T_{\perp}^{(0)}=p_{\perp}^{(1)} / p_{\perp}^{(0)}-\rho^{(1)} / \rho^{(0)}$. It follows that 
$\Pi_{x x}=-\Pi_{y y}=n^{(0)} m \int\left(v_{\perp}^{2} / 2\right) f^{(1)} \cos 2 \phi d^{3} v, \Pi_{z z}=0$, $\Pi_{x y}=n^{(0)} m \int\left(v_{\perp}^{2} / 2\right) f^{(1)} \sin 2 \phi d^{3} v_{,} \Pi_{x z}=n^{(0)} m \int v_{\|} v_{\perp}$ $v_{\|} v_{\perp} f^{(1)} \cos \phi d^{3} v+\left(p_{\perp}^{(0)}-p_{\|}^{(0)}\right) \widehat{b}_{x}$ and $\Pi_{y z}=n^{(0)} m \int$ $v_{\|} v_{\perp} f^{(1)} \sin \phi d^{3} v+\left(p_{\perp}^{(0)}-p_{\|}^{(0)}\right) \widehat{b}_{y}$. Some technical details concerning the asymptotic calculation of the above quantities are given in Appendix A. We concentrate here on the resulting expressions.

\subsubsection{Parallel and Transverse Temperatures}

[31] Neglecting contributions of the order of $\theta^{2}=k_{z}^{2} / k_{\perp}^{2}$, as justified by the discussion in section 3 , and thus retaining the leading order only, one gets for the parallel and transverse temperatures of the ions

$$
\begin{aligned}
\frac{T_{\| p}^{(1)}}{T_{\| p}^{(0)}}= & \left(1-R\left(\zeta_{p}\right)+2 \zeta_{p}^{2} R\left(\zeta_{p}\right)\right) \frac{T_{\perp p}^{(0)}}{T_{\| p}^{(0)}} \\
& \times\left[\left(\Gamma_{1}(b)-\Gamma_{0}(b)\right) \frac{b_{z}}{B_{0}}-\Gamma_{0}(b) \frac{e \Psi}{T_{\perp p}^{(0)}}\right]
\end{aligned}
$$

and

$$
\begin{aligned}
\frac{T_{\perp p}^{(1)}}{T_{\perp p}^{(0)}}= & \left(\frac{T_{\perp p}^{(0)}}{T_{\| p}^{(0)}} R\left(\zeta_{p}\right)-1\right) \\
& \times\left(-2 b \Gamma_{1}(b)+2 b \Gamma_{0}(b)-\Gamma_{0}(b)\right) \frac{b_{z}}{B_{0}} \\
& -\left(b \Gamma_{1}(b)-b \Gamma_{0}(b)\right) R\left(\zeta_{p}\right) \frac{e \Psi}{T_{\| p}^{(0)}} \\
& +\left(b \Gamma_{1}(b)-b \Gamma_{0}(b)\right) \frac{e}{T_{\perp p}^{(0)}}\left(\Phi+\frac{k_{z}^{2}}{k_{\perp}^{2}}(\Phi-\Psi)\right)
\end{aligned}
$$

Analogous expressions are obtained for the electrons, with the functions $\Gamma(b)$ then taken in the $b=0$ limit.

\subsubsection{Gyroviscous Stress}

[32] The kinetic theory gives

$$
\begin{aligned}
\frac{\Pi_{x x}}{p_{\perp p}^{(0)}}= & -\cos 2 \psi\left(b \Gamma_{0}(b)-\Gamma_{1}(b)-b \Gamma_{1}(b)\right) \\
& \times\left[2\left(\frac{T_{\perp p}^{(0)}}{T_{\| p}^{(0)}} R\left(\zeta_{p}\right)-1\right) \frac{b_{z}}{B_{0}}+R\left(\zeta_{p}\right) \frac{e \Psi}{T_{\| p}^{(0)}}\right. \\
& \left.-\frac{e}{T_{\perp p}^{(0)}}\left(\Phi+\frac{k_{z}^{2}}{k_{\perp}^{2}}(\Phi-\Psi)\right)\right] \\
& -\cos 2 \psi \Gamma_{1}(b)\left(\frac{T_{\perp p}^{(0)}}{T_{\| p}^{(0)}} R\left(\zeta_{p}\right)-1\right) \frac{b_{z}}{B_{0}}+i \sin 2 \psi \frac{k_{z}}{\Omega} \frac{k_{z}}{\omega} \\
& \times \frac{e}{T_{\perp p}^{(0)}}\left[\left(\Gamma_{0}(b)-\Gamma_{1}(b)\right)-\frac{1}{b}\left(1-\Gamma_{0}(b)\right)\right] \\
& \times\left[\frac{T_{\perp p}^{(0)}-T_{\| p}^{(0)}\left(1+\frac{k_{z}^{2}}{k_{\perp}^{2}}\right)(\Phi-\Psi)}{m_{p}}\right. \\
& \left.-\frac{\omega^{2}}{k_{z}^{2}}\left(\Phi+\frac{k_{z}^{2}}{k_{\perp}^{2}}(\Phi-\Psi)\right)\right] \\
& -i \sin 2 \psi \frac{\omega}{\Omega}\left[\frac{1}{b}\left(\Gamma_{0}(b)-1-\Gamma_{1}(b)\right)\right. \\
& \left.+2\left(\Gamma_{0}(b)-\Gamma_{1}(b)\right)\right] \frac{b_{z}}{B_{0}}, \\
&
\end{aligned}
$$

together with

$$
\begin{aligned}
\frac{\Pi_{x z}}{p_{\| p}^{(0)}}= & i \sin \psi \frac{T_{\perp}^{(0)}}{T_{\|}^{(0)}} \frac{k_{\perp}}{\Omega} \frac{\omega}{k_{z}}\left(\Gamma_{0}(b)-\Gamma_{1}(b)\right) \\
& \times R(\zeta)\left(2 \frac{T_{\perp}^{(0)}}{T_{\|}^{(0)}} \frac{b_{z}}{B_{0}}+\frac{e \Psi}{T_{\|}^{(0)}}\right)+\cos \psi \frac{k_{z}}{k_{\perp}}\left(\frac{T_{\perp}^{(0)}}{T_{\|}^{(0)}}-1\right) \\
& \times\left[\left(\Gamma_{0}(b)-\Gamma_{1}(b)-1\right) \frac{b_{z}}{B_{0}}-\left(1-\Gamma_{0}(b)\right) \frac{e \Psi}{T_{\perp}^{(0)}}\right] \\
& -\left[\cos \psi\left(\frac{T_{\perp}^{(0)}}{T_{\|}^{(0)}}-2\right) \frac{k_{z}}{k_{\perp}}\left(1-\Gamma_{0}(b)\right)\right. \\
& +i \sin \psi \frac{T_{\perp}^{(0)}-T_{\|}^{(0)}}{m} \frac{T_{\perp}^{(0)}}{T_{\|}^{(0)}} \frac{k_{z} k_{\perp}}{\omega \Omega} \\
& \left.\times\left(\Gamma_{0}(b)-\Gamma_{1}(b)-1\right)\right]\left(1+\frac{k_{z}^{2}}{k_{\perp}^{2}}\right) \frac{e}{T_{\perp}^{(0)}}(\Phi-\Psi) \\
& +4 i C_{1}^{3}(b) \sin \psi\left(\frac{T_{\perp}^{(0)}}{T_{\|}^{(0)}}-2\right) \frac{k_{z} \omega}{k_{\perp} \Omega} \frac{b_{z}}{B_{0}}
\end{aligned}
$$

The elements $\Pi_{x y}$ and $\Pi_{y z}$ are deduced from $\Pi_{x x}$ and $\Pi_{x x}$ respectively, by replacing $\sin 2 \psi$ by $-\cos 2 \psi$ and $\cos 2 \psi$ by $\sin 2 \psi$. Furthermore, the contributions involving $\Phi-\Psi$ are conveniently expressed in terms of the transverse magnetic field components by using equations (4) and (5).

\subsection{Modeling of the Temperatures}

[33] The MHD description of anisotropic plasmas often involves the double adiabatic law of Chew et al. [1956], that relates the fluctuations of the gyrotropic pressure components to those of the density and of the magnetic field intensity through universal power law dependencies. The relevance of such a functional dependency with adjustable exponents was recently stressed by Stasiewicz [2005], who suggested empirical fittings on the basis of the analysis of observational data provided by the Cluster spacecraft mission, for a broad range of values of the plasma $\beta$. An approach based on the use of complex polytropic indices calculated as function of the mode properties is presented by Belmont et al. [1992]. The analysis is based on the fluid hierarchy for the lowest order moments, supplemented by the kinetic expression of the polytropic indices provided by the kinetic theory as derived by Belmont and Mazelle [1992].

[34] In this section, we determine the temperatures of the various species in a form that accurately reproduces the linear kinetic theory.

[35] When remaining at the level of the linear approximation, one can simply write

$$
\begin{gathered}
T_{\| r}=T_{\| r}^{(0)}\left(1+\alpha_{\| r}\right) \\
T_{\perp r}=T_{\perp r}^{(0)}\left(1-A_{r} \frac{b_{z}}{B_{0}}+\alpha_{\perp r}\right),
\end{gathered}
$$

where the contributions denoted by $\alpha_{\| r}$ and $\alpha_{\perp r}$ are specified below using linearized kinetic theory. This model is mostly adapted to the quasi-isothermal dynamics near the 
threshold of the mirror instability. Observations show that the magnetosheath plasma does not strongly depart from an isothermal behavior [Phan et al., 1994]. The term $-A_{r} b_{z} / B_{0}$ has been singled out in equation (25) as it originates from the linearization of a more general equation of state obtained in a quasi-static fluid description. This leads to define nonlinear generalizations of the above formulas by replacing $1-A_{r} b_{z} /$ $B_{0}$ by an appropriate function whose detailed form does not affect the expression of the quantities $\alpha_{\| r}$ and $\alpha_{\perp r}$.

[36] A straightforward but somehow arbitrary nonlinear extension can be obtained in a power law form as in the double-polytropic model of Hau and Sonnerup [1993], used by Baumgärtel [2001]. In the framework of our model, the exponents are given by $\gamma_{\| r}=1$ and $\gamma_{\perp r}=1-A_{r}$ when using the notation of these authors.

[37] More precise formulae consistent with a slowdynamics ordering [Ramos, 2005] can also be derived. In contrast with the adiabatic limit, the behavior of the gyrotropic pressures in the quasi-static regime is prescribed by the equations for the parallel and perpendicular (gyrotropic) heat fluxes [Chust and Belmont, 2006]. This behavior is not universal and depends on the distribution function. When neglecting the deviation from bi-Maxwellian distribution functions, a quasi-normal closure can be performed. Balancing, in this quasi-static large-scale limit, the dominant terms in the equations governing the parallel and perpendicular heat fluxes (equations (42), (43) and (53) of Goswami et al. [2005] or equations (69) and (70) of Ramos [2005]), yields for each particle species

$$
\begin{gathered}
\partial_{\|} T_{\| r}=0 \\
\frac{\partial_{\|} T_{\perp r}}{T_{\perp r}}=\left(1-\frac{T_{\perp r}}{T_{\| r}}\right) \frac{\partial_{\|}|B|}{|B|},
\end{gathered}
$$

a system also given by Chust and Belmont [2006]. The parallel temperatures are constant and the perpendicular ones are given by

$$
T_{\perp r}=T_{\perp r}^{(0)} \frac{|B|}{(A+1) B \mid-A B_{0}} .
$$

Note that an additional equation, associated with the longitudinal force balance, leads to [see, e.g., Goldston and Rutherford, 2000]

$$
q_{r} n_{r} E_{\|}+\partial_{\|} p_{\| r}+\left(p_{\perp r}-p_{\| r}\right) \frac{\partial_{\|}|B|}{|B|}=0
$$

For cold electrons, $E_{\|}=0$. As a consequence, combining equations (27) and (29), one gets for the protons

$$
\frac{n}{n^{(0)}}=\frac{T_{\perp p}}{T_{\perp p}^{(0)}}
$$

It is then interesting to notice that after using equation (30), equation (28) coincides with the relation for the upper value $B_{+}$of the magnetic field in the kinetic model of Pantellini [1998].

[38] Equation (28) can also be obtained by a Lagrangian integration of the Vlasov equation, assuming stationarity and bi-Maxwellian distributions at a boundary [Chust and Belmont, 2006]. Note that the above equation implies a lower bound for the magnetic field minima in a time independent regime, namely $|B| / B_{0}>A /(A+1)$. This constraint, which is not stringent in the weakly nonlinear regime, can be modified when retaining deviations from biMaxwellians, whose evaluation is outside the scope of the present paper. It turns out that such corrections do not essentially affect the parallel temperatures.

[39] Let us now characterize $\alpha_{\perp r}$ and $\alpha_{\| r}$. Using equation (20) for the parallel temperatures, one has for the ions

$$
\begin{aligned}
\alpha_{\| p}= & \frac{T_{\perp p}^{(0)}}{T_{\| p}^{(0)}}\left(1-R\left(\zeta_{p}\right)+2 \zeta_{p}^{2} R\left(\zeta_{p}\right)\right) \\
& \times\left[\left(\Gamma_{1}(b)-\Gamma_{0}(b)\right) \frac{b_{z}}{B_{0}}-\Gamma_{0}(b) \frac{e \Psi}{T_{\perp p}^{(0)}}\right]
\end{aligned}
$$

and for the electrons

$$
\alpha_{\| e}=-\frac{T_{\perp e}^{(0)}}{T_{\| e}^{(0)}}\left(1-R\left(\zeta_{e}\right)+2 \zeta_{e}^{2} R\left(\zeta_{e}\right)\right)\left(\frac{b_{z}}{B_{0}}+\frac{e \Psi}{T_{\perp e}^{(0)}}\right) .
$$

[40] In order to eliminate the plasma response function from the above formulas, it is convenient to introduce the hydrodynamic velocities of each species along the ambient field. This gives

$$
\begin{aligned}
\alpha_{\| p}= & \frac{1-R\left(\zeta_{p}\right)+2 \zeta_{p}^{2} R\left(\zeta_{p}\right)}{\operatorname{sgn}\left(k_{z}\right) \zeta_{p} R\left(\zeta_{p}\right)} \sqrt{\frac{m_{p}}{2 T_{\| p}^{(0)}}} \\
& \cdot\left[u_{z p}+\frac{T_{\perp p}^{(0)}-T_{\| p}^{(0)}}{m_{p}} \frac{1-\Gamma_{0}(b)}{b} \frac{1}{v_{A}^{2}} \frac{j_{z}}{e n^{(0)}}\right] \\
\alpha_{\| e}= & \frac{1-R\left(\zeta_{e}\right)+2 \zeta_{e}^{2} R\left(\zeta_{e}\right)}{\operatorname{sgn}\left(k_{z}\right) \zeta_{e} R\left(\zeta_{e}\right)} \sqrt{\frac{m_{e}}{2 T_{\| e}^{(0)}}}\left[u_{z e}-\frac{T_{\perp e}^{(0)}-T_{\| e}^{(0)}}{m_{p}} \frac{1}{v_{A}^{2}} \frac{j_{z}}{e n^{(0)}}\right] .
\end{aligned}
$$

[41] At this step, the plasma response function is to be replaced by a Padé approximant. If, for the sake of simplicity, one chooses a two-pole approximation of the plasma response function, one gets $\left(1-R+2 \zeta^{2} R\right) /\left[\operatorname{sgn}\left(k_{z}\right) \zeta R\right] \approx$ $-i \sqrt{\pi} k_{z} /\left|k_{z}\right|$ that keeps a finite value when $\zeta$ becomes infinite, which is not satisfactory. One easily checks that reproducing the proper decay of the Landau damping in this adiabatic limit, actually requires the use of at least the threepole approximant $R_{3}(\zeta)=\frac{2-i \sqrt{\pi} \zeta}{2-3 i \sqrt{\pi} \zeta-4 \zeta^{2}+2 i \sqrt{\pi} \zeta^{3}}$, which yields $\frac{1-R(\zeta)+2 \zeta_{i}^{2} R(\zeta)}{\zeta R(\zeta)} \approx \frac{2 i \sqrt{\pi}}{-2+i \sqrt{\pi} \zeta}$, whose imaginary part does tend to zero as $\zeta \rightarrow \infty$. Substituting in [equations (33) and (34)] and returning to physical space for the time and longitudinal coordinate variables, we are led to prescribe $\alpha_{\| p}$ and $\alpha_{\| e}$ as the solutions of the dynamical equations

$$
\begin{aligned}
\left(\partial_{t}\right. & \left.-\frac{2}{\sqrt{\pi}} \sqrt{\frac{2 T_{\| p}^{(0)}}{m_{p}}} \mathcal{H}_{z} \partial_{z}\right) \alpha_{\| p} \\
& +2 \partial_{z}\left[u_{z p}+\frac{T_{\perp p}^{(0)}-T_{\| p}^{(0)}}{m_{p}} \frac{1-\Gamma_{0}(b)}{b} \frac{1}{v_{A}^{2}} \frac{j_{z}}{e n^{(0)}}\right]=0
\end{aligned}
$$




$$
\begin{aligned}
\left(\partial_{t}\right. & \left.-\frac{2}{\sqrt{\pi}} \sqrt{\frac{2 T_{\| e}^{(0)}}{m_{e}}} \mathcal{H}_{z} \partial_{z}\right) \alpha_{\| e} \\
& +2 \partial_{z}\left[u_{z e}-\frac{T_{\perp e}^{(0)}}{m_{p}} \frac{1}{v_{A}^{2}} \frac{j_{z}}{e n^{(0)}}\right]=0
\end{aligned}
$$

where $\mathcal{H}_{z}$ denotes the Hilbert transform relatively to the $\mathrm{z}$ variable.

[42] Turning to the transverse quantities, we write

$$
\begin{aligned}
\alpha_{\perp p}= & b\left(\Gamma_{0}(b)-\Gamma_{1}(b)\right)\left[2\left(\frac{T_{\perp p}^{(0)}}{T_{\| i}^{(0)}} R\left(\zeta_{p}\right)-1\right) \frac{b_{z}}{B_{0}}\right. \\
& \left.+R\left(\zeta_{p}\right) \frac{e \Psi}{T_{\| i}^{(0)}}-\frac{e}{T_{\perp i}^{(0)}}\left(\Phi+\frac{k_{z}^{2}}{k_{\perp}^{2}}(\Phi-\Psi)\right)\right] \\
& +\left[A_{p}-\Gamma_{0}(b)\left(\frac{T_{\perp p}^{(0)}}{T_{\| p}^{(0)}} R\left(\zeta_{p}\right)-1\right)\right] \frac{b_{z}}{B_{0}}
\end{aligned}
$$

By comparison with the kinetic expression for $\chi_{s p}$, we get

$$
\alpha_{\perp p}+\Gamma_{0}(b) \frac{T_{\perp p}^{(0)}}{T_{\| p}^{(0)}} R\left(\zeta_{p}\right) \frac{b_{z}}{B_{0}}=\frac{k_{\perp}^{2}}{\Omega} \chi_{s p}+K_{p} \frac{b_{z}}{B_{0}}
$$

with

$$
K_{p}=4 C_{1}^{3}(b) \frac{T_{\perp p}^{(0)}-T_{\| p}^{(0)}}{m_{p}} \frac{k_{z}^{2}}{\Omega^{2}}+A_{p}+\Gamma_{0}(b)
$$

[43] In the case of $\alpha_{\perp p}$, the isothermal and adiabatic limits are correctly reproduced by replacing the plasma response function by its one pole approximation $R_{1}\left(\zeta_{p}\right)=1$ / $\left(1-i \sqrt{\pi} \zeta_{p}\right)$. This description appears to be sufficient and leads to the dynamical equation

$$
\begin{gathered}
{\left[\partial_{t}-\frac{1}{\sqrt{\pi}} \sqrt{\frac{2 T_{\| p}^{(0)}}{m_{p}}} \mathcal{H}_{z} \partial_{z}\right]\left(\alpha_{\perp p}-\frac{k_{\perp}^{2}}{\Omega} \chi_{s p}-K_{p} \frac{b_{z}}{B_{0}}\right)} \\
-\frac{1}{\sqrt{\pi}} \sqrt{\frac{2 T_{\| p}^{(0)}}{m_{p}} \Gamma_{0}(b) \frac{T_{\perp p}^{(0)}}{T_{\| p}^{(0)}} \mathcal{H}_{z} \partial_{z} \frac{b_{z}}{B_{0}}=0 .}
\end{gathered}
$$

Note that $k_{\perp}^{2} \chi_{s p}$ identifies with the longitudinal vorticity $\omega_{z p}=\widehat{z} \cdot\left(\nabla \times u_{p}\right)$ of the proton flow.

[44] The equivalent equation for the electrons is simply

$$
\begin{gathered}
{\left[\partial_{t}-\frac{1}{\sqrt{\pi}} \sqrt{\frac{2 T_{\| e}^{(0)}}{m_{e}}} \mathcal{H}_{z} \partial_{z}\right]\left(\alpha_{\perp e}-\left(A_{e}+1\right) \frac{b_{z}}{B_{0}}\right)} \\
-\frac{1}{\sqrt{\pi}} \sqrt{\frac{2 T_{\| e}^{(0)}}{m_{e}}} \frac{T_{\perp e}^{(0)}}{T_{\| e}^{(0)}} \mathcal{H}_{z} \partial_{z} \frac{b_{z}}{B_{0}}=0 .
\end{gathered}
$$

In the above equation, $\partial_{t} b_{z}$ is expressed using the FaradayMaxwell equation and the generalized Ohm's law.

[45] The above closure equations were derived by a linear analysis. In order to restore Galilean invariance, it may nevertheless be suitable to replace the partial time derivatives $\partial_{t}$ acting on the $\alpha$ 's by the convective derivative $\partial_{t}+u \cdot \nabla$ of the associated particle species.

\subsection{Modeling of the Gyroviscous Stress}

[46] It is convenient to write

$$
\frac{1}{p_{\perp p}^{(0)}} \nabla_{\perp} \cdot \Pi_{\perp}=-\nabla_{\perp} \mathcal{A}+\nabla_{\perp} \times(\mathcal{B} \hat{z}) .
$$

The quantities $\mathcal{A}$ and $\mathcal{B}$ are expressed by means of equation (22). At this level, we check that, to leading order in $k_{z}^{2} / k_{\perp}^{2}$, $\mathcal{A}$ and $\mathcal{B}$ reduce to the quantities $-\delta p_{c}$ and $-\delta p_{s}$ of $C h e n g$ and Johnson [1999], when assuming a zero drift frequency.

[47] Using equation (13) with the $\mathcal{S}_{3}$ correction neglected, together with equation (21) that gives

$$
\begin{aligned}
& \left(\frac{T_{\perp p}^{(0)}}{T_{\| p}^{(0)}} R\left(\zeta_{p}\right)-1\right) \frac{b_{z}}{B_{0}}=\frac{1}{\Gamma_{0}(b)} \\
& \quad \times\left(\frac{k_{\perp}^{2}}{\Omega} \chi_{s p}+4 \frac{T_{\perp p}^{(0)}-T_{\| p}^{(0)}}{m_{p}} \frac{k_{z}^{2}}{\Omega^{2}} C_{1}^{3}(b) \frac{b_{z}}{B_{0}}-\frac{T_{\perp p}^{(1)}}{T_{\perp p}^{(0)}}\right),
\end{aligned}
$$

we obtain

$$
\begin{aligned}
\mathcal{A}= & \left(1-\frac{\Gamma_{1}(b)}{b\left[\Gamma_{0}(b)-\Gamma_{1}(b)\right]}+\frac{\Gamma_{1}(b)}{\Gamma_{0}(b)}\right) \frac{k_{\perp}^{2}}{\Omega} \chi_{s p}-\frac{\Gamma_{1}(b)}{\Gamma_{0}(b)} \frac{T_{\perp p}^{(1)}}{T_{\perp p}^{(0)}} \\
\mathcal{B}= & -i \frac{\omega}{\Omega}\left[\frac{\Gamma_{0}(b)-1-\Gamma_{1}(b)}{b}+2\left(\Gamma_{0}(b)-\Gamma_{1}(b)\right)\right. \\
& \left.+\frac{\Gamma_{0}(b)-\Gamma_{1}(b)}{1-\Gamma_{0}(b)}\left(\Gamma_{0}(b)-\Gamma_{1}(b)-\frac{1-\Gamma_{0}(b)}{b}\right)\right] \frac{b_{z}}{B_{0}} \\
& +\frac{1}{1-\Gamma_{0}(b)}\left[\Gamma_{0}(b)-\Gamma_{1}(b)-\frac{1-\Gamma_{0}(b)}{b}\right] \frac{k_{\perp}^{2}}{\Omega} \chi_{c p} .
\end{aligned}
$$

In $\mathcal{A}$, we have neglected a contribution of the form $4 C_{1}^{3}(b)\left(\frac{b \Gamma_{0}(b)-\Gamma_{1}(b)-b \Gamma_{1}(b)}{\Gamma_{0}(b)-\Gamma_{1}(b)}+\frac{b \Gamma_{1}(b)}{\Gamma_{0}(b)}\right) \frac{T_{\perp p}^{(0)}-T_{\| p}^{(0)}}{T_{\perp p}^{(0)}}$ $\frac{k_{z}^{2}}{k_{\perp}^{2}} \frac{b_{z}}{B_{0}}$ that, without being totally negligible, is nevertheless relatively small due to the factor $k_{z}^{2} / k_{\perp}^{2}$. We check that this term has essentially no effect at the level of the dispersion relation and can thus be discarded in the fluid model. The quantity $i \omega b_{z} / B_{0}$ is as previously estimated using the Faraday-Maxwell equation and the contributions $\chi_{s p}$ and $\chi_{c p}$ are given by $\chi_{s p} \widehat{z}=\left(i k_{\perp} \times u_{\perp p}\right) / k_{\perp}^{2}$ and $\chi_{c p}=\left(i k_{\perp}\right.$. $\left.u_{\perp p}\right) / k_{\perp}^{2}$.

[48] Let us now turn to $\Pi_{z}=\left(\Pi_{x z}, \Pi_{y z}, \Pi_{z z}\right)$ where $\Pi_{z z}=0$ in the linear description. This vector was neglected by Smolyakov et al. [1995] and Cheng and Johnson [1999], but turns out to be significant. Writing

$$
\Pi_{z}=-\nabla_{\perp} \mathcal{C}+\nabla_{\perp} \times(\mathcal{D} \widehat{z})
$$

simplified expressions for $\mathcal{C}$ and $\mathcal{D}$ can be derived from equation (23) by noticing that the contributions involving $\left(\frac{T_{\perp p}^{(0)}}{T_{\| p}^{(0)}}-2\right) \frac{k_{z}}{k_{\perp}}\left(1-\Gamma_{0}(b)\right)\left(1+\frac{k_{z}^{2}}{k_{\perp}^{2}}\right) \frac{e}{T_{\perp p}^{(0)}}(\Phi-\Psi)$ in 
equation (23) and in the corresponding equation for $\Pi_{y z}$ are small and also that neglecting the terms proportional to $\omega$ has a minor influence at the level of the dispersion relation. A more accurate description is possible but would lead to a much more cumbersome formalism. Making the above approximations, we are led to write

$$
\begin{aligned}
\frac{\mathcal{C}}{p_{\| p}^{(0)}}= & i \frac{k_{z}}{k_{\perp}^{2}}\left(\frac{T_{\perp p}^{(0)}}{T_{\| p}^{(0)}}-1\right)\left[\left(\Gamma_{0}(b)-\Gamma_{1}(b)-1\right) \frac{b_{z}}{B_{0}}\right. \\
& \left.-\left(1-\Gamma_{0}(b)\right) \frac{e \Psi}{T_{\perp p}^{(0)}}\right] \\
\frac{\mathcal{D}}{p_{\| p}^{(0)}} & =\left(\Gamma_{0}(b)-\Gamma_{1}(b)-1\right)\left(\frac{T_{\perp p}^{(0)}}{T_{\| p}^{(0)}}-1\right) \frac{4 \pi}{c B_{0} k_{\perp}^{2}} j_{z} .
\end{aligned}
$$

\subsection{Comparison With the Kinetic Theory}

[49] In order to test the accuracy of the Landau fluid model, we compare in this section its predictions for the dispersion relation of various MHD waves, and for the growth rate of the mirror instability, with the results of the full kinetic theory reported in section 2. Linearizing the fluid model in a reference frame where $\partial / \partial y=0$, one easily derives

$$
\begin{gathered}
-\partial_{t} \partial_{x x} \chi_{c p}+\partial_{x x}\left(\frac{p_{\perp}}{\rho_{p}^{(0)}}-\frac{p_{\perp}^{(0)}}{\rho_{p}^{(0)}} \mathcal{A}+c_{A}^{2} \frac{b_{z}}{B_{0}}\right) \\
+\left(c_{A}^{2}+\frac{p_{\perp}^{(0)}-p_{\|}^{(0)}}{\rho_{p}^{(0)}}\right) \partial_{z z} \frac{b_{z}}{B_{0}}-\frac{p_{\| p}^{(0)}}{\rho_{p}^{(0)}} \partial_{x x z} \mathcal{C}=0 \\
-\partial_{t} \partial_{x x} \chi_{s p}-\left(c_{A}^{2}+\frac{p_{\perp}^{(0)}-p_{\|}^{(0)}}{\rho_{p}^{(0)}}\right) \partial_{x z} \frac{b_{y}}{B_{0}} \\
-\frac{p_{\perp}^{(0)}}{\rho_{p}^{(0)}} \partial_{x x} \mathcal{B}-\frac{p_{\|}^{(0)}}{\rho_{p}^{(0)}} \partial_{x x z} \mathcal{D}=0 \\
\partial_{t} u_{z p}+\partial_{z}\left(\frac{p_{\|}}{\rho_{p}^{(0)}}+\frac{p_{\perp}^{(0)}-p_{\|}^{(0)}}{\rho_{p}^{(0)}} \frac{b_{z}}{B_{0}}\right)-\frac{p_{\| p}^{(0)}}{\rho_{p}^{(0)}} \partial_{x x} \mathcal{C}=0,
\end{gathered}
$$

where the pressures without subscript denote the sum of the proton and electron pressures and where we have used the generalized Ohm's law that rewrites

$$
\begin{gathered}
\frac{c}{B_{0}} E_{x}=\partial_{x} \chi_{s p}-\frac{c_{A}^{2}}{\Omega} \partial_{x} \frac{b_{z}}{B_{0}} \\
+\left(\frac{c_{A}^{2}}{\Omega}+\frac{p_{\perp e}^{(0)}-p_{\| e}^{(0)}}{\rho_{p}^{(0)} \Omega}\right) \partial_{z} \frac{b_{x}}{B_{0}}-\frac{1}{\rho_{p}^{(0)} \Omega} \partial_{x} p_{\perp e} \\
\frac{c}{B_{0}} E_{y}=-\partial_{x} \chi_{c p}+\left(\frac{c_{A}^{2}}{\Omega}+\frac{p_{\perp e}^{(0)}-p_{\| e}^{(0)}}{\rho_{p}^{(0)} \Omega}\right) \partial_{z} \frac{b_{y}}{B_{0}} \\
\Psi=\frac{1}{n_{0} e}\left(p_{\| e}+\left(p_{\perp e}^{(0)}-p_{\| e}^{(0)}\right) \frac{b_{z}}{B_{0}}\right) .
\end{gathered}
$$

We also have

$$
\begin{gathered}
\partial_{t} \frac{n_{p}}{n_{0}}-\partial_{x x} \chi_{c p}+\partial_{z} u_{z p}=0 \\
\partial_{t} \frac{b_{y}}{B_{0}}+\frac{c}{B_{0}}\left(\partial_{z} E_{x}+\partial_{x z} \Psi\right)=0 \\
\partial_{t} \frac{b_{z}}{B_{0}}+\frac{c}{B_{0}} \partial_{x} E_{y}=0
\end{gathered}
$$

together with the relations $\partial_{x} b_{x}+\partial_{z} b_{z}=0$ and $j_{z}=\frac{c}{4 \pi} \partial_{x} b_{y}$ [50] Equations (48)-(50) and (54)-(56), supplemented by the expressions for the pressures resulting from equations (35)-(36) and equations (39)-(40), provide a closed system from which the dispersion relation is easily obtained using a symbolic calculator.

[51] In order to test the capability of the model to describe KAWs, we first address the asymptotic regime of low $\beta_{\perp p}$ with electrons much hotter than ions considered in section 3.1. For this purpose, we supplement Figure 1 that displays the frequency of these waves as a function of perpendicular wavenumber, with the prediction of the fluid model (cross symbols). We observe that the agreement between the model and the kinetic theory remains satisfactory in all the displayed range of scales $(b<0.19)$, thus much beyond the validity domain of the explicit asymptotic formula.

[52] With the aim to consider plasmas with higher $\beta$ where the Landau damping is more efficient, we compare the predictions of the fluid model and of the kinetic theory using $\beta_{\perp p}=1$ with equal and isotropic equilibrium temperatures for the ions and the electrons $(\tau=1)$, keeping the direction parameter $\theta=10^{-3}$. As seen on Figure 5, the dispersion relation is very accurately reproduced by the model up to about $b=0.15$. At larger transverse wavenumber, the simplification we performed by neglecting the term involving $\partial_{t} b_{y}$ in $\Pi_{x z}$ is no longer possible and should be restored to preserve accuracy.

[53] Concerning magneto-sonic waves propagating perpendicular to the ambient magnetic field, the phase velocity is well reproduced by the model, since the adiabatic regime associated with the condition $\partial_{t} \gg \partial_{z}$ is correctly described by the dynamical equations (35)-(36) and (39)-(40). The dispersive corrections in the wavenumber cone $k_{z} / k_{\perp} \ll 1$ which require second order accuracy in a $1 / \Omega$ expansion of the FLR terms, are however not properly captured. The kinetic theory developed in the previous section is also insufficient to describe this finite frequency mode that requires higher order terms in the development of the quantities $X_{\gamma}$ and $Y_{\gamma}$ defined in Appendix A. In fact, only a few terms proportional to $\omega^{4} / \Omega^{4}$ are to be calculated, that originate from the next order contribution to $X_{0}$, the other extra terms in $Y_{0}, X_{1}$ and $Y_{1}$ being all proportional to $k_{z}$. These terms give rise to an extra contribution to $n_{p}^{(1)} / n_{p}^{(0)}$ that reads $\frac{2}{b}\left(b \Gamma_{0}(b)-b \Gamma_{1}(b)-\Gamma_{1}(b)\right) \frac{\omega^{4}}{\Omega^{4}} \frac{b_{z}}{B_{0}}$ and to an extra contribution to $u_{y p}$ (taking the angle $\psi=0$ ) given by $-2 i \sqrt{\frac{2 T_{\perp p}^{(0)}}{m_{p}}} \sqrt{\frac{2}{b}}\left(b \Gamma_{1}(b)-b \Gamma_{0}(b)+\Gamma_{1}(b)+\frac{\Gamma_{1}(b)}{2 b}\right) \frac{\omega^{4}}{\Omega^{4}} \frac{b_{z}}{B_{0}}$. 


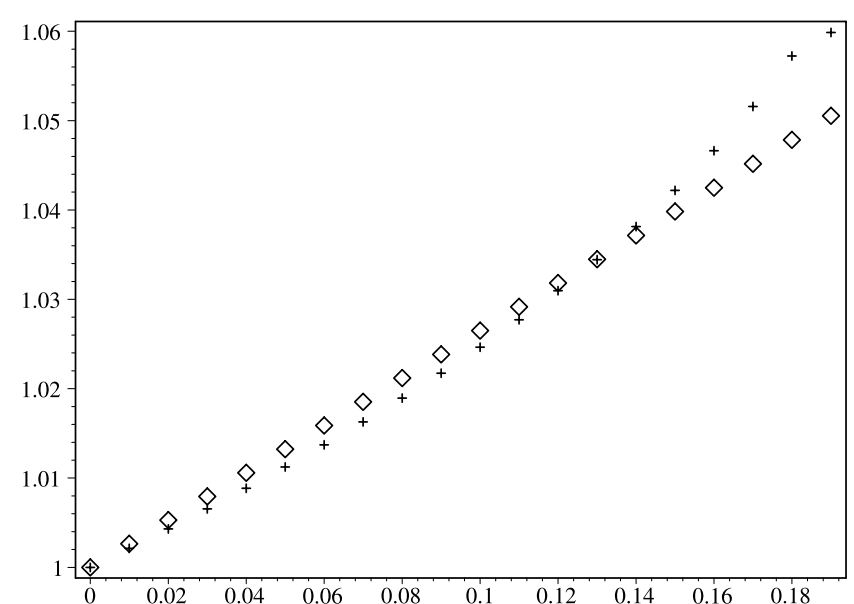

Figure 5. Comparison of the normalized frequencies $\Re(\omega) / k_{z} v_{A}$ of KAWs as a function of $b$ for $\theta=10^{-3}$, $\beta_{\perp p}=1, \tau=1$ and isotropic equilibrium temperatures, obtained by numerical resolution of the full dispersion relation (diamonds) and from the fluid model described in section 4 (crosses).

The integrals are here calculated keeping only the $l= \pm 1$ terms in the summation of equation (A1). Additional terms are to be added as $b$ is increased, but their contribution does not exceed a few percents when $b=O(1)$. With these extra terms, one easily computes the dispersion relation of transverse magnetosonic waves and verifies its agreement with equations (2.10) - (2.11) of Mikhailovskii and Smolyakov [1985]. Figure 6 displays $\Re(\omega) / k_{\perp} v_{A}$ as a function of $b$ for $\beta_{\| p}=1, \tau=1$ and $A_{p}=A_{e}=0$ (taking $\theta=10^{-4}$ ) as resulting from the numerical resolution of the dispersion relation of the full kinetic theory (diamonds), from the analytic formula of Mikhailovskii and Smolyakov [1985] (crosses), which is only valid for very small values of $b$, and from the fluid model (circles). As announced, for transverse magnetosonic waves, the fluid model is only valid at the point $b=0$.

[54] We now turn to the main property of this model, i.e. its capability to model mirror modes at finite values of the parameter $b$. We first address the case of cold electrons and parameters corresponding to a regime close to the mirror instability threshold $\left(A_{p}=0.7, \beta_{\perp p}=1.5, \theta=0.1\right)$. As seen on Figure 7 , the agreement between the kinetic theory (diamonds) and the fluid model (circles) is excellent, except at the largest values of the parameter $b$ for which a deviation is visible, although small. In order to analyze the origin of the discrepancy at these small scales, we symbolize by crosses the results obtained when the dispersion relation is derived from the fluid model in an improved form that retains all terms in the nongyrotropic pressures and a fourth pole approximation used for the plasma response function in the first term of $\Pi_{y z}$. Note that these extra terms are not easily incorporated in a fluid model suitable to address initial value problems, due to the presence of high powers of the frequency in their denominator. They are only used here in the computation of the dispersion relation for comparison. Figure 7 shows that the agreement between kinetic theory and this "extended" fluid model is then excellent throughout the entire $b$-range. This suggests that a proper modeling of these extra terms in a more sophisticated model, would ensure a high accuracy up to the smallest transverse scales.

[55] For parameters corresponding to a finite distance to threshold and finite electron temperature $\left(\tau=1, A_{p}=1.5\right.$, $A_{e}=0.1, \beta_{\perp p}=1.5, \theta=0.2$, we compare in Figure 8 the results of the kinetic theory (diamonds) with those of the fluid model (circles) for the imaginary part of the frequency as a function of $k_{\perp} r_{p}$. Crosses again correspond to the "extended" fluid model. As seen on this graph, the agreement between kinetic theory and the fluid model is good (and even better when including the extra terms) when the growth rate takes small values. It clearly deteriorates close to the maximum growth rate as the conditions for the validity of the asymptotics performed on the kinetic theory are violated. The important point is that the model reproduces the large-scale behavior with asymptotic accuracy and displays the correct qualitative behavior when $b$ is of order unity.

[56] As already noted, in the above regime where the proton $\beta$ is of order unity, the mirror instability may be subdominant compared with the AIC instability. In order to validate the model in a regime where the mirror instability is dominant, we also considered the case where $\beta=5, \tau=1$, $A_{e}=0$ and a pressure anisotropy $A_{p}=0.5$. The value $\theta=0.1$ was retained for the direction parameter. As seen in Figure 9, a satisfactory accuracy is provided by the fluid model for the growth rate of the unstable modes, the decay rate of the damped modes being less precisely reproduced. As also seen on the figure, retaining the full nongyrotropic pressures is necessary to insure a uniform accuracy.

\section{Concluding Remarks}

[57] We have constructed a Landau fluid model that retains small-scale FLR effects in order to reproduce the growth rate of the mirror instability for perturbations with arbitrary transverse wavenumber, and in particular the arrest of the instability for perturbations at small enough scales.

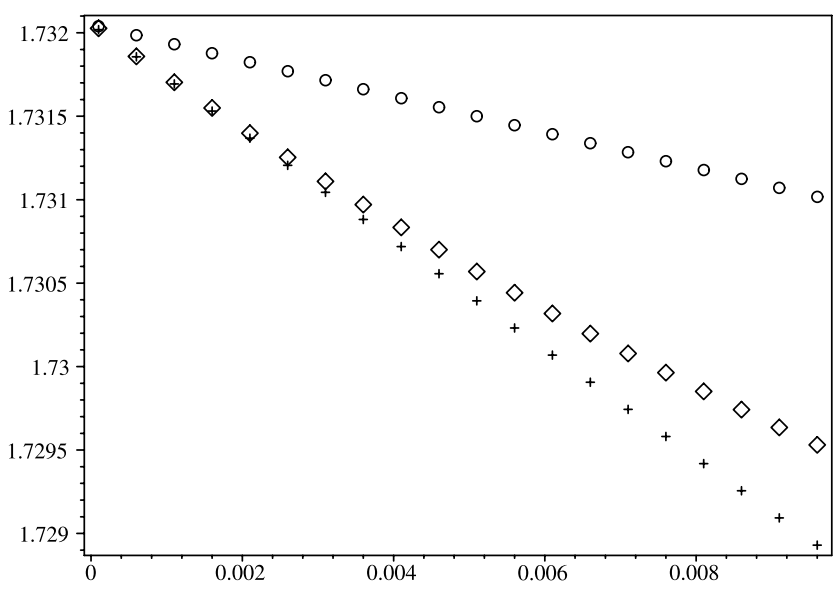

Figure 6. Normalized frequencies $\Re(\omega) / k_{\perp} v_{A}$ of transverse magnetosonic waves as a function of $b$ for $\beta_{\perp p}=1$, $\tau=1$ and isotropic equilibrium temperatures, given by numerical resolution of the fluid model (circles), the full kinetic theory (diamond) and by the asymptotic formula of Mikhailovskii and Smolyakov [1985] (crosses). 


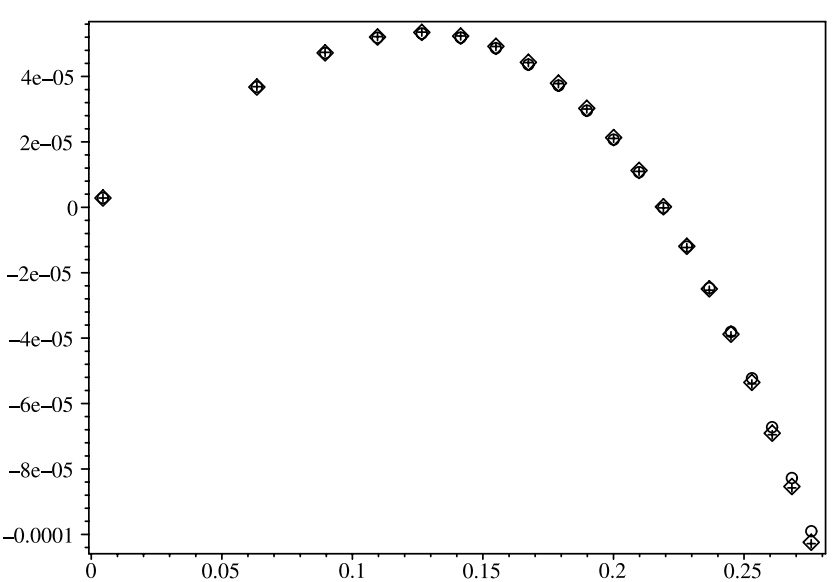

Figure 7. Mirror mode growth rates $\gamma / \Omega$ as a function of $k_{\perp} r_{p}=\sqrt{2 b}$ for $\tau=0, A_{p}=0.7, \beta_{\perp p}=1.5, \theta=0.1$ obtained from kinetic theory (diamonds) and the fluid model (circles). Crosses correspond to an extended version of the model (see text).

This latter property is indeed a prerequisite for a model to be mathematically well posed in regimes where a mirror instability develops. The present model should provide an efficient tool to simulate the quasi-transverse dynamics in the weakly nonlinear regime, and in particular reproduce the formation and evolution of coherent structures and powerlaw spectra, as observed in the terrestrial magnetosheath [Sahraoui et al., 2003; Tsurutani et al., 2005]. Such regimes seem indeed beyond the possibility of the present days fully kinetic simulations, at least in three space dimensions. Their study using the present fluid model will be the object of a forthcoming paper. It is interesting to note at this point that, although oversimplified, the model used by Baumgärtel [2001] demonstrates that significant aspects of the mirror mode dynamics can be captured within a fluid approach. Our model that correctly arrests the mirror mode instability

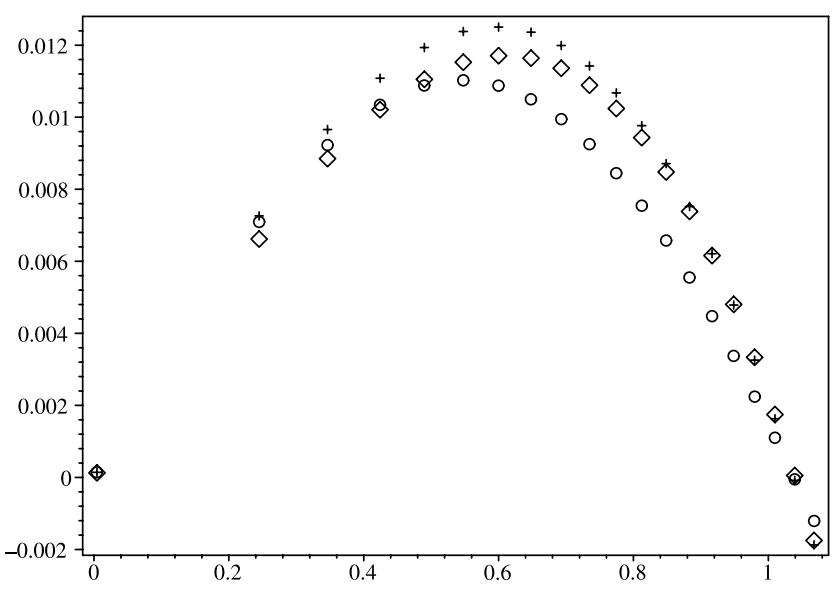

Figure 8. Mirror mode growth rates $\gamma / \Omega$ as a function of $k_{\perp} r_{p}=\sqrt{2 b}$ for $\tau=1, A_{p}=1.5, A_{e}=0.1, \beta_{\perp p}=1.5, \theta=0.2$ obtained from kinetic theory (diamonds) and the fluid model (circles). Crosses correspond to a linear model where all terms are kept in the FLR corrections and a fourth pole approximation is used for the plasma response function in the first term of $\Pi_{y z}$. at small scales by the effect of Landau damping and finite Larmor radius corrections, should provide a quantitative improvement to this description.

[58] The present model is however not asymptotically exact. It is based on a procedure consisting in describing nonlinear interactions within a fluid formalism and restricting kinetic effects to a linear approximation. These kinetic corrections are expressed in terms of usual hydrodynamic variables by deriving from the kinetic theory specific relations between the fluid moments, and replacing in the resulting relations, the plasma response function by Padé approximants, suitably chosen to ensure asymptotic accuracy in the limits where the parallel phase velocities are asymptotically small or large compared with the parallel thermal velocities of the ions. This procedure results in closure relations in the form of a few dynamical equations amenable to numerical integration. A similar development is reported by Goswami et al. [2005] in the context of a high order Landau fluid closure for the large-scale dynamics.

[59] The observation that the present model quantitatively reproduces the instability growth rate of the mirror modes at all scales, suggests that it captures at least part of the dynamics of the so-called trapped particles that play an important role in the analysis of Kivelson and Southwood [1996] and Pantellini [1998]. In this context it is of interest to note that suppressing the imaginary part of $\alpha_{\| p}$ and $\alpha_{\perp p}$ (associated with Landau damping) significantly affects the instability growth rate (its value is multiplied by a factor 2 in the conditions of Figure 9) and also suppresses the damping of the modes that are not unstable. In contrast, the electron Landau damping has no significant effects and could be neglected, as usually done in hybrid simulations.

[60] Direct validations of the model in the nonlinear regime by comparisons with fully kinetic simulations are in project. Further improvement are possible, but at the price of a significant increase of its complexity, by retaining fully nonlinear equations for the gyrotropic pressures and heat fluxes, and closing the hierarchy at the level of fourth

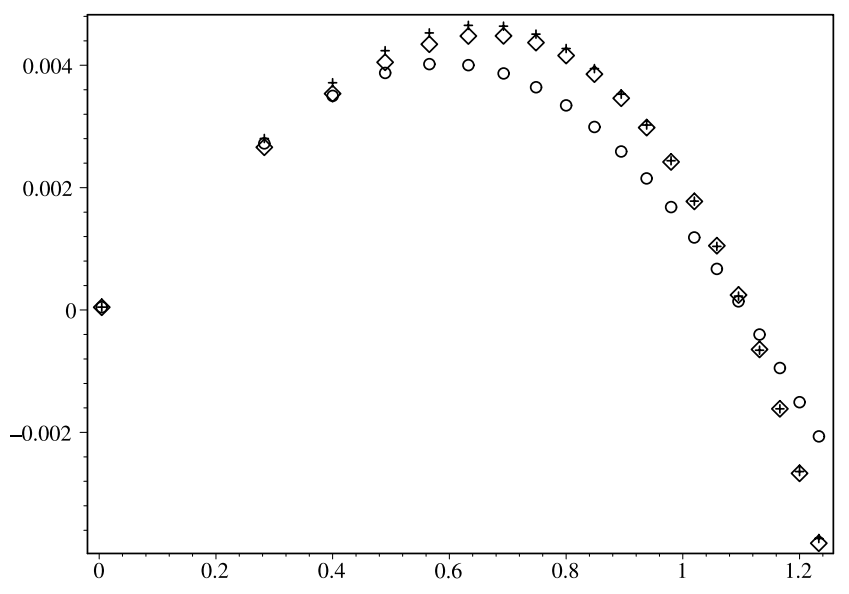

Figure 9. Mirror mode growth rates $\gamma / \Omega$ as a function of $k_{\perp} r_{p}=\sqrt{2 b}$ for $\tau=1, A_{p}=1.5, A_{e}=0, \beta_{\perp p}=5, \theta=0.1$ obtained from kinetic theory (diamonds), from the fluid model (circles) and from an improved model where all terms are kept in the FLR corrections and a fourth pole approximation is used for the plasma response function in the first term of $\Pi_{y z}$ (crosses). 
order cumulants. Such a development is necessary for the model to accurately describe oblique Alfvén and magnetosonic waves, as illustrated by Passot and Sulem [2004a, 2004b] and Goswami et al. [2005]. Matching in this case the fluid description to the kinetic theory (as needed to capture the dynamics at small transverse scales) is however a delicate issue that is presently under investigation.

\section{Appendix A}

[61] In order to evaluate the velocities and density fluctuations needed to derive the dispersion relation, we compute the first moments of $f^{(1)}$ as expansions in powers of the ratio $\omega / \Omega$, assuming $\omega / \Omega \ll 1$ with no condition on the magnitude of $\left(k_{\perp} / \Omega\right) \sqrt{2 T_{\perp}^{(0)} / m}$ nor on that of $\omega / k_{z}$. This expansion is carried out to second order. We concentrate the analysis on the ions and, in order to simplify the writing, now suppress the subscript $p$.

[62] Nondimensional velocities and potentials are introduced by writing $v_{\perp}=\widetilde{v}_{\perp} \sqrt{2 T_{\perp}^{(0)} / m}, v_{\|}=\widetilde{v}_{\|} \sqrt{2 T_{\|}^{(0)} / m}$, $\Phi=\widetilde{\Phi} T_{\perp}^{(0)} / e, \Psi=\widetilde{\Psi} T_{\perp}^{(0)} / e, b_{z}=B_{0} \widetilde{b}_{z}$. One also defines $\alpha=\left(k_{\perp} / \Omega\right) \sqrt{2 T_{\perp}^{(0)} / m}$, and $\zeta_{l}=\left[(\omega-l \Omega) /\left|k_{z}\right|\right] \sqrt{m / 2 T_{\|}^{(0)}}$. The quantity $\zeta_{0}$ will simply be denoted by $\zeta$. The computation of the hydrodynamic moments [number density $n=n \int f d^{3} v$, velocity $u=\int v f d^{3} v / \int f d^{3} v$, pressure tensor $\mathbf{p}=m n \int(v-u)$ $\left.\otimes(v-u) f d^{3} v\right]$ then involves the estimate of integrals of the form ( $\beta$ and $\gamma$ are here nonnegative integers)

$$
\begin{gathered}
\int e^{i p \phi} f^{(1)} v_{\|}^{\gamma} v_{\perp}^{\beta+1} d v_{\perp} d v_{\|} d \phi=2\left(\frac{2 T_{\|}^{(0)}}{m}\right)^{\frac{\gamma}{2}}\left(\frac{2 T_{\perp}^{(0)}}{m}\right)^{\frac{\beta}{2}} \\
\cdot e^{i p \psi} \int \sum_{l=-\infty}^{+\infty}\left\{\sqrt{\frac{2}{b}} J_{l}^{\prime}\left(\alpha \widetilde{v}_{\perp}\right) J_{l-p}\left(\alpha \widetilde{v}_{\perp}\right) \widetilde{v}_{\perp} X_{\gamma}\left(\zeta_{l}\right) \widetilde{b}_{z}\right. \\
-J_{l}\left(\alpha \widetilde{v}_{\perp}\right) J_{l-p}\left(\alpha \widetilde{v}_{\perp}\right) \frac{l \Omega}{\omega} X_{\gamma}\left(\zeta_{l}\right)\left(\widetilde{\Phi}+\frac{k_{z}^{2}}{k_{\perp}^{2}}(\widetilde{\Phi}-\widetilde{\Psi})\right) \\
\left.-Y_{\gamma}\left(\zeta_{l}\right) J_{l}\left(\alpha \widetilde{v}_{\perp}\right) J_{l-p}\left(\alpha \widetilde{v}_{\perp}\right)\left[\frac{T_{\perp}^{(0)}}{T_{\|}^{(0)}}-\frac{l \Omega}{\omega}\left(\frac{T_{\perp}^{(0)}}{T_{\|}^{(0)}}-1\right)\right] \widetilde{\Psi}\right\} \\
\cdot e^{-\widetilde{v}_{\perp}{ }^{2} \widetilde{v}_{\perp}{ }^{\beta+1} d \widetilde{v}_{\perp} .}
\end{gathered}
$$

We use the notation

$$
X_{\gamma}\left(\zeta_{l}\right)=\zeta Y_{\gamma-1}\left(\zeta_{l}\right)+\left(\frac{T_{\perp}^{(0)}}{T_{\|}^{(0)}}-1\right) Y_{\gamma}\left(\zeta_{l}\right)
$$

where $Y_{\gamma}\left(\zeta_{l}\right)$ is the analytic continuation on the real axis of the function defined by $\frac{1}{\sqrt{\pi}} \int_{-\infty}^{+\infty} \frac{e^{-x^{2}} x^{\gamma+1}}{x-\zeta_{l}} d x$ for $\operatorname{Im} \zeta>0$. Introducing the plasma dispersion function $Z(\zeta)=\frac{1}{\sqrt{\pi}}$ $P \int_{-\infty}^{+\infty} \frac{e^{-x^{2}}}{x-\zeta} d x+i \sqrt{\pi} e^{-\zeta 2}$ and the plasma response function $R(\zeta)=1+\zeta Z(\zeta)$, one has $Y_{0}\left(\zeta_{l}\right)=R\left(\zeta_{l}\right)$ and $Y_{1}\left(\zeta_{l}\right)=$ $\zeta_{l} R\left(\zeta_{l}\right)$

[63] It is convenient in the sum involved in the right hand side of equation (A1) to distinguish the contribution of $l=0$ that leads to a singular term, from the contributions of $l \neq 0$.
The computation of the velocities and the density fluctuations of the various species require the asymptotic estimate of $X_{j}\left(\zeta_{l}\right)$ and $Y_{j}\left(\zeta_{l}\right)$ when $l \neq 1$ for $j=0$ and 1 . In this cases, one write $\zeta_{l}=-\zeta \frac{l \Omega}{\omega}\left(1-\frac{\omega}{l \Omega}\right)$. Using that $Z(\zeta)=-1 / \zeta-1 / 2 \zeta^{3}-$ $3 / 4 \zeta^{5}+O\left(1 / \zeta^{7}\right)$ for $\zeta \rightarrow+\infty$, one obtains when expanding at the order $(\omega / \Omega)^{3}$ needed to get a uniform description of the moments at order $(\omega / \Omega)^{2}$,

$$
\begin{aligned}
X_{0}\left(\zeta_{l}\right)= & \frac{\omega}{l \Omega}+\left[1-\frac{1}{2 \zeta_{0}^{2}}\left(\frac{T_{\perp}^{(0)}}{T_{\|}^{(0)}}-1\right)\right]\left(\frac{\omega}{l \Omega}\right)^{2} \\
& +\left[1-\frac{1}{\zeta_{0}^{2}}\left(\frac{T_{\perp}^{(0)}}{T_{\|}^{(0)}}-\frac{3}{2}\right)\right]\left(\frac{\omega}{l \Omega}\right)^{3} \\
X_{1}\left(\zeta_{l}\right)= & \frac{1}{2 \zeta_{0}}\left(\frac{T_{\perp}^{(0)}}{T_{\|}^{(0)}}-1\right)\left(\frac{\omega}{l \Omega}\right)+\frac{1}{2 \zeta_{0}}\left(\frac{T_{\perp}^{(0)}}{T_{\|}^{(0)}}-2\right)\left(\frac{\omega}{l \Omega}\right)^{2} \\
& +\left[\frac{1}{2 \zeta_{0}}\left(\frac{T_{\perp}^{(0)}}{T_{\|}^{(0)}}-3\right)+\frac{3}{4 \zeta_{0}^{3}}\left(\frac{T_{\perp}^{(0)}}{T_{\|}^{(0)}}-1\right)\right]\left(\frac{\omega}{l \Omega}\right)^{3} \\
Y_{1}\left(\zeta_{l}\right)= & \frac{1}{2 \zeta_{0}}\left(\frac{\omega}{\Omega}\right)+\frac{1}{\zeta_{0}^{2}}\left(\frac{\omega}{l \Omega}\right)^{3} \\
2 \zeta_{0} & \left(\frac{\omega}{l \Omega}\right)^{2}+\left(\frac{1}{2 \zeta_{0}}+\frac{3}{4 \zeta_{0}^{3}}\right)\left(\frac{\omega}{l \Omega}\right)^{3} .
\end{aligned}
$$

We are thus led to sum series of the form

$$
S_{p}^{k}(x)=\sum_{l \neq 0} \frac{1}{l^{k}} J_{l}(x) J_{l-p}(x)
$$

and

$$
\Sigma_{p}^{k}(x)=\sum_{l \neq 0} \frac{1}{l^{k}} J_{l}^{\prime}(x) J_{l-p}(x)
$$

and to define the integrals

$$
C_{s}^{l}(b)=\int \widetilde{v}_{\perp}^{l} \Sigma_{s}^{2}\left(\alpha \widetilde{v}_{\perp}\right) e^{-\widetilde{v}_{\perp}^{2}} d \widetilde{v}_{\perp}
$$

and

$$
D_{s}^{l}(b)=\int \widetilde{v}_{\perp}^{l} S_{s}^{2}\left(\alpha \widetilde{v}_{\perp}\right) e^{-\widetilde{v}_{\perp}^{2}} d \widetilde{v}_{\perp}
$$

[64] One has $S_{0}^{0}(x)=1-J_{0}^{2}(x), S_{0}^{1}(x)=0, \Sigma_{0}^{1}(x)=0$, $S_{1}^{0}(x)=-S_{-1}^{0}=J_{0}(x) J_{1}(x), S_{1}^{1}(x)=S_{-1}^{1}=\frac{1}{x}\left(1-J_{0}^{2}(x)\right)$ and $\Sigma_{1}^{1}(x)$ $\Sigma_{1}^{1}(x)=\Sigma_{-1}^{1}=\frac{1}{x} J_{0}(x) J_{1}(x)$, and one also makes use of the identities $\int_{0}^{+\infty} J_{0}^{2}(\alpha x) e^{-x 2} \frac{x d x}{\sqrt{2 b}}=\frac{1}{2} e^{-b} I_{0}(b)$, $\int_{0}^{+\infty} J_{0}(\alpha x) J_{0}^{\prime}(\alpha x) e^{-x 2} x^{2} d x=\frac{\sqrt{2 b}}{4} e^{-b}\left(I_{1}(b)-I_{0}(b)\right)$, $\int_{0}^{+\infty} J_{1}^{2}(\alpha x) e^{-x 2} x^{3} d x=\frac{b}{2} e^{-b}\left(I_{0}(b)-I_{1}(b)\right)$, where $I_{\nu}(b)$ is 
the modified Bessel function of order $\nu$. It is thus convenient to introduce the standard notation $\Gamma_{\nu}(b)=e^{-b} I_{\nu}(b)$.

[65] The series $S_{p}^{k}(x)$ and $\Sigma_{p}^{k}(x)$ for $k \geq 2$ are in contrast difficult to compute exactly but can be estimated with a sufficient accuracy by retaining only the contributions originating from $l= \pm 1$ and in some cases from $l= \pm 2$ also. In the expression of $u_{z p}$, we approximate $C_{0}^{2}(b)$ by retaining the contributions of $l= \pm 1$ and $l= \pm 2$ that are comparable, in the form $C_{0}^{2}(b) \approx(1 / 4 \sqrt{2 b})\left(3 b \Gamma_{0}(b)-3 \Gamma_{1}(b)-2 \Gamma_{1}(b)-\right.$ $\left.2 \Gamma_{0}(b)+4 \Gamma_{1}(b) / b\right)$. For $D_{0}^{1}(b)$, we only retain the contribution of $l= \pm 1$, which gives $D_{0}^{1}(b) \approx \Gamma_{1}(b)$. Further contributions are conveniently computed using a symbolic calculator. The contribution of $l= \pm 2$ gives for example $\left(b \Gamma_{0}(b)-2 \Gamma_{1}(b)\right) /(4 b)$. One then checks that such corrections do not significantly affect the summation and can be overlooked for the sake of simplicity. Using a similar approximation for coefficients entering $\chi_{p s}$, we approximate $C_{1}^{3}(b) \approx\left(-3 b \Gamma_{0}(b)+2 \Gamma_{0}(b)+3 b \Gamma_{1}(b)+2 \Gamma_{1}(b)-2 \Gamma_{1}(b) / b+\right.$ $\left.2 \Gamma_{0}(b) / b-4 \Gamma_{1}(b) / b^{2}\right) / 4$ and $D_{1}^{2}(b) \approx(1 / \sqrt{2 b})\left(b \Gamma_{0}(b)-\right.$ $\left.b \Gamma_{1}(b)-\Gamma_{1}(b)\right)$.

[66] The computation of the pressure tensor components in the low-frequency limit requires the additional computation of

$$
\begin{array}{r}
X_{2}\left(\zeta_{l}\right)=\frac{1}{2}\left(\frac{\omega}{l \Omega}\right)+\left[\frac{1}{2}-\frac{3}{4 \zeta_{0}^{2}}\left(\frac{T_{\perp}^{(0)}}{T_{\|}^{(0)}}-1\right)\right]\left(\frac{\omega}{l \Omega}\right)^{2} \\
+\left[\frac{1}{2}-\frac{3}{\zeta_{0}^{2}}\left(\frac{T_{\perp}^{(0)}}{T_{\|}^{(0)}}-\frac{3}{2}\right)\right]\left(\frac{\omega}{l \Omega}\right)^{3} \\
Y_{2}\left(\zeta_{l}\right)=-\frac{3}{4 \zeta_{0}^{2}}\left(\frac{\omega}{l \Omega}\right)^{2}-\frac{3}{2 \zeta_{0}^{2}}\left(\frac{\omega}{l \Omega}\right)^{3} .
\end{array}
$$

One also needs the identities

$$
\begin{gathered}
S_{2}^{1}(x)=S_{-2}^{1}=-J_{0}(x) J_{2}(x) \\
S_{2}^{2}(x)=-S_{-2}^{2}=\frac{2}{x} J_{0}(x) J_{1}(x)-\frac{2}{x^{2}}\left(1-J_{0}^{2}(x)\right) \\
S_{2}^{3}(x)=-S_{-2}^{3}=-\frac{2}{x^{2}} J_{0}(x) J_{1}(x)+\frac{1}{x}\left(1-2 J_{1}^{2}(x)\right)
\end{gathered}
$$

and

$$
\begin{aligned}
& \int_{0}^{+\infty} J_{0}^{2}(\alpha x) e^{-x^{2}} x^{3} d x=-\frac{1}{2} e^{-b}\left(b I_{0}(b)-I_{0}(b)-b I_{1}(b)\right) \\
& \int_{0}^{+\infty} J_{2}(\alpha x) J_{0}(\alpha x) e^{-x^{2}} x^{3} d x=\frac{1}{2} e^{-b}\left(b I_{0}(b)-I_{1}(b)-b I_{1}(b)\right) .
\end{aligned}
$$

\section{Appendix B}

[67] The contributions $\mathcal{S}_{1}, \mathcal{S}_{2}$ and $\mathcal{S}_{3}$ arising in equations (8), (10) and (13) are given by

$$
\begin{aligned}
& \mathcal{S}_{1}=-2 \sqrt{\frac{2 m_{p}}{T_{\perp p}^{(0)}}} \frac{T_{\perp p}^{(0)}-T_{\| p}^{(0)}}{m_{p}} \frac{k_{z}^{2}}{k_{\perp} \Omega} C_{0}^{2}(b) \frac{b_{z}}{B_{0}} \\
& +2 \frac{k_{z}^{2}}{\Omega^{2}} \frac{2 T_{\perp p}^{(0)}-3 T_{\mid p}^{(0)}}{m_{p}} D_{0}^{1}(b) \frac{e}{T_{\perp p}^{(0)}}\left(\Phi+\frac{k_{z}^{2}}{k_{\perp}^{2}}(\Phi-\Psi)\right) \\
& -2 \frac{k_{z}^{2}}{\Omega^{2}} \frac{T_{\perp p}^{(0)}-2 T_{\| p}^{(0)}}{m_{p}} D_{0}^{1}(b) \frac{e}{T_{\perp p}^{(0)}} \Psi+2 \sqrt{\frac{2 m_{p}}{T_{\perp p}^{(0)}}} C_{0}^{2}(b) \frac{\omega^{2}}{k_{\perp} \Omega} \frac{b_{z}}{B_{0}} \\
& -2 D_{0}^{1}(b) \frac{\omega^{2}}{\Omega^{2}} \frac{e}{T_{\perp p}^{(0)}}\left(\Phi+\frac{k_{z}^{2}}{k_{\perp}^{2}}(\Phi-\Psi)\right) .
\end{aligned}
$$

$$
\begin{aligned}
\mathcal{S}_{2}= & -\frac{k_{z}}{\omega} \frac{T_{\perp p}^{(0)}-T_{\| p}^{(0)}}{m_{p}}\left(1-\Gamma_{0}(b)\right) \frac{e}{T_{\perp p}^{(0)}}\left(1+\frac{k_{z}^{2}}{k_{\perp}^{2}}\right)(\Phi-\Psi) \\
& +2 \sqrt{\frac{2 T_{\|}^{(0)}}{m}} \sqrt{\frac{T_{\|}^{(0)}}{T_{\perp}^{(0)}}\left(\frac{T_{\perp}^{(0)}}{T_{\|}^{(0)}}-2\right) C_{0}^{2}(b) \frac{k_{z} \omega}{k_{\perp} \Omega} \frac{b_{z}}{B_{0}}} \\
& -D_{0}^{1}(b)\left[2 \frac{T_{\| p}^{(0)}}{m_{p}}\left(\frac{T_{\perp p}^{(0)}}{T_{\| p}^{(0)}}-3\right) \frac{k_{z} \omega}{\Omega^{2}}\right. \\
& \left.+6\left(\frac{T_{\| p}^{(0)}}{m}\right)^{2}\left(\frac{T_{\perp p}^{(0)}}{T_{\| p}^{(0)}}-1\right) \frac{k_{z}^{3}}{\omega \Omega^{2}}\right] \frac{e}{T_{\perp p}^{(0)}}\left(\Phi+\frac{k_{z}^{2}}{k_{\perp}^{2}}(\Phi-\Psi)\right) \\
& +D_{0}^{1}(b)\left[-2 \frac{T_{\| p}^{(0)}}{m} \frac{k_{z} \omega}{\Omega^{2}}+6\left(\frac{T_{\| p}^{(0)}}{m}\right)^{2}\left(\frac{T_{\perp p}^{(0)}}{T_{\| p}^{(0)}}-1\right) \frac{k_{z}^{3}}{\omega \Omega^{2}}\right] \frac{e}{T_{\perp p}^{(0)}} \Psi .
\end{aligned}
$$

$$
\begin{aligned}
\mathcal{S}_{3}= & 2 \sqrt{\frac{2 T_{\perp p}^{(0)}}{m_{p}} \frac{k_{z}^{2}}{k_{\perp} \Omega^{2}}} \\
& \times \frac{2 T_{\perp p}^{(0)}-3 T_{\| p}^{(0)}}{m_{p}} D_{1}^{2}(b) \frac{e}{T_{\perp p}^{(0)}}\left(\Phi+\frac{k_{z}^{2}}{k_{\perp}^{2}}(\Phi-\Psi)\right) \\
& -2 \sqrt{\frac{2 T_{\perp p}^{(0)}}{m_{p}}} \frac{k_{z}^{2}}{k_{\perp} \Omega^{2}} \frac{T_{\perp p}^{(0)}-2 T_{\| p}^{(0)}}{m_{p}} D_{1}^{2}(b) \frac{e}{T_{\perp p}^{(0)}} \Psi \\
& +4 C_{1}^{3}(b) \frac{\omega^{2}}{k_{\perp}^{2} \Omega} \frac{b_{z}}{B_{0}}-2 \sqrt{\frac{2 T_{\perp p}^{(0)}}{m_{p}}} D_{1}^{2}(b) \frac{\omega^{2}}{k_{\perp} \Omega^{2}}\left(\Phi+\frac{k_{z}^{2}}{k_{\perp}^{2}}(\Phi-\Psi)\right) .
\end{aligned}
$$

[68] Acknowledgments. We acknowledge useful discussions with G. Belmont, T. Chust, and F. Sahraoui. This work benefited from support of the CNRS program "Soleil-Terre."

[69] Shadia Rifai Habbal thanks Filippo G. Pantellini, Steven J Schwartz, and another referee for their assistance in evaluating this paper.

\section{References}

Akhiezer, A. I., I. A. Akhiezer, R. V. Polovin, A. G. Sitenko, and K. N. Stepanov (1975), Plasma Electrodynamics, vol. 1, Elsevier, New York. 
Baumgärtel, K. (2001), Fluid approach to mirror mode structures, Planet. Space Sci., 49, 1239.

Belmont, G., and C. Mazelle (1992), Polytropic indices in collisionless plasmas: Theory and measurements, J. Geophys. Res., 97(A6), 8327.

Belmont, G., D. Hubert, C. Lacombe, and F. Pantellini (1992), Mirror mode and other compressible modes, in Proceedings of the 26th ESLAB Symposium-Study of the Solar-Terrestrial System, (Killarney, Ireland 16-19 June 1992), ESA Spec. Publ., SP-346, Sept.

Bugnon, G., R. Goswami, T. Passot, and P. L. Sulem (2006), Towards fluid simulations of dispersive MHD waves in a warm collisionless plasma, Adv. Space Res., in press.

Chandrasekhar, S. A., A. N. Kaufman, and K. M. Watson (1958), The stability if the pinch, Proc. R. Soc. London, Ser. A, 245, 435.

Cheng, C. Z., and J. R. Johnson (1999), A kinetic-fluid model, J. Geophys. Res., 104(A1), 413

Chew, G. F., M. L. Goldberger, and F. E. Low (1956), Boltzmann equation and the one-fluid hydrodynamic equations in the absence of particle collisions, Proc. R. Soc. (London), Ser. A, 236, 21,611.

Chust, T., and G. Belmont (2006), Closure of fluid equations in collsionless magnetoplasmas, Phys. Plasmas, 11, 012506.

Gary, S. P. (1992), The mirror an ion cyclotron anisotropy instabilites, J. Geophys. Res., 97(A6), 8519

Gary, S. P., B. J. Anderson, R. E. Denton, S. A. Fuselier, and M. E. McKean (1994), A limited closure relation for anisotropic plasmas from the Earth's magnetosheath, Phys. Plasmas, 1, 1676.

Génot, V., S. J. Schwartz, C. Mazelle, M. Balikin, M. Dunlop, and T. M. Bauer (2001), Kinetic study of the mirror mode, J. Geophys. Res., 106(A10), 413.

Goldston, R. J., and P. H. Rutherford (2000), Introduction to Plasma Physics, 2nd ed., Inst. of Phys. Publ., Philadelphia, $\mathrm{Pa}$

Goswami, P., T. Passot, and P. L. Sulem (2005), A Landau fluid model for warm collisionless plasmas, Phys. Plasmas, 12, 102, 109.

Hasegawa, A., and L. Chen (1975), Kinetic process of plasma heating due to Alfvén wave excitation, Phys. Rev. Lett., 35, 370.

Hasegawa, A., and L. Chen (1976), Kinetic processes in plasma heating by resonant mode conversion of Alfvén wave, Phys. Fluids, 19, 1924.

Hau, L. N., and B. U. O. Sonnerup (1993), On slow-mode waves in an anisotropic plasma, Geophys. Res. Lett., 20, 1763.

Huddleston, D. E., R. J. Strangeway, X. Blanco-Cano, C. T. Russel, M. G. Kivelson, and K. K. Khurana (1999), Mirror mode structures at the Gallileo-Ito flyby: Instability criterion and dispersion analysis, J. Geophys. Res., 104(A8), 17,479.

Kaufman, R. L., J. T. Horng, and A. Wolfe (1970), Large amplitude hydromagnetic waves in the inner magnetosheath, J. Geophys. Res., 75, 4666

Kivelson, M. G., and D. J. Southwood (1996), Mirror instability II: The mechanism of nonlinear saturation, J. Geophys. Res., 101(A8), 17,365.

Lacombe, C., F. G. E. Pantellini, D. Hubert, C. C. Harvey, A. Mangeney, G. Belmont, and C. T. Russel (1992), Mirror and Alfvénic waves observed by ISEE 1-2 during crossings of the Earth's bow shock, Ann. Geophys., 10,772 .

Leckband, J. A., D. Burgess, F. G. E. Pantellini, and S. J. Schwartz (1995), Ion distributions associated with mirror waves in the earth's magnetosheath, Adv. Space Res., 15(8/9), 345

Lin, C.-H., J. K. Chao, L. C. Lee, D. J. Wu, Y. Li, B. H. Wu, and P. Song (1998), Identification of mirror waves by the phase difference between perturbed magnetic field and plasmas, J. Geophys. Res., 103(A4), 6621

Lucek, E. A., M. W. Dunlop, A. Balogh, P. Cargill, W. Baumjoham, E. Georgescu, G. Haerendel, and K. H. Fornacon (1999), Mirror mode structures observed in the dawn-side magnetosheath by Equator-S, Geophys. Res. Lett., 26, 2159.

Lucek, E. A., M. W. Dunlop, T. S. Horbury, A. Balogh, P. Brown, P. Cargill, C. Carr, K. H. Fornacon, E. Georgescu, and T. Oddy (2001), Cluster magnetic field observations in the magnetosheath: Four point measurements of mirror structures, Ann. Geophys., 19, 1421.

McKean, M. E., P. Gary, and D. Winske (1992), Mirror and ion cyclotron anisotropy instabilities in the magnetosphere, J. Geophys. Res., 97(A12), 19,421 .

McKean, M. E., S. P. Gary, and D. Winske (1993), Kinetic physics of the mirror instability, J. Geophys. Res., 98(A12), 21,313.

McKean, M. E., D. Winske, and S. P. Gary (1994), Two-dimensional simulations of the anisotropy instabilities in the magnetosheath, J. Geophys. Res., 99(A6), 11,141.

McKean, M. E., N. Omidi, and D. Krauss-Varban (1995), Wave and ion evolution downstream of quasi-perpendicular bow shocks, J. Geophys. Res., 100(A3), 3427.
Mikhailovskii, A. B., and A. I. Smolyakov (1985), Theory of low-frequency magnetosonic solitons, Sov. Phys. JETP, 61, 109.

Pantellini, F. G. E. (1998), A model of the formation of stable nonpropagating magnetic structures in the solar wind based on the nonlinear mirror instability, J. Geophys. Res., 103(A3), 4789

Pantellini, F. G. E., and S. J. Schwartz (1995), Electron temperature effects in the linear proton mirror instability, J. Geophys. Res., 100(A3), 3539.

Pantellini, F. G. E., D. Burgess, and S. J. Schwartz (1995), On nonlinear mirror instability, Adv. Space Res., 15, 341.

Passot, T., and P. L. Sulem (2004a), A fluid description for Landau damping of dispersive MHD waves, Nonlin. Processes Geophys., 11, 245.

Passot, T., and P. L. Sulem (2004b), A Landau fluid model for dispersive magnetohydrodynamics, Phys. Plasmas, 11, 5173.

Phan, T. D., G. Paschmann, W. Baumjohann, N. Sckopke, and H. Lühr (1994), The magnetosheath region adjacent to the dayside magnetopause: AMPTE/IRM observations, J. Geophys. Res., 99(A1), 121.

Pokhotelov, O. A., M. A. Balikhin, H. St.-C. K. Alleyne, and O. G. Onishchenko (2000), Mirror instability with finite electron temperature, J. Geophys. Res., 105(A2), 2393.

Pokhotelov, O. A., R. Z. Sagdeev, M. A. Balikhin, and R. A. Treumann (2004), Mirror instability at finite-Larmor radius wavelengths, J. Geophys. Res., 109, A09213, doi:10.1029/2004JA010568.

Ramos, J. J. (2005), Fluid formalism for collisionless magnetized plasmas, Phys. Plasmas, 12, 052, 102.

Russel, C. T., D. E. Huddleston, R. J. Strangeway, X. Blanco-Cano, M. G. Kivelson, K. K. Khurana, L. A. Frank, W. Paterson, D. A. Gurnett, and W. S. Kurth (1999), Mirror-mode structures at the Galileo-Ito flyby: Observations, J. Geophys. Res., 104(A8), 17,471.

Sahraoui, F., et al. (2003), ULF wave identification in the magnetosheath: The k-filtering technique applied to Cluster II data, J. Geophys. Res., 108(A9), 1335, doi:10.1029/2002JA009587.

Sahraoui, F., G. Belmont, J. L. Pinçon, L. Rezeau, A. Balogh, P. Robert, and N. Cornilleau-Wehrlin (2004), Magnetic turbulent spectra in the magnetosheath: New insights, Ann. Geophys., 22, 2283, S-Ref-ID: 1432-0576/ag/2004-22-2283

Sahraoui, F., G. Belmont, L. Rezeau, J. L. Pinçon, N. Cornilleau-Wehrlin, and A. Balog (2006), Solar wind-Earth coupling: Turbulent fragmentation of magnetic structures seen by Cluster, Phys. Rev. Lett., 96, 075002.

Schwartz, S. J., D. Burgess, and J. J. Moses (1996), Low-frequency waves in the Earth's magnetosheath: Present status, Ann. Geophys., 14, 1134.

Sckopke, N., G. Paschmann, A. L. Brinca, C. W. Carlson, and H. Lühr (1990), Ion thermalization in quasi-perpendicular shocks involving reflected ions, J. Geophys. Res., 95(A5), 63,337.

Smolyakov, A. I., I. O. Pogutse, and A. Hirose (1995), Fluid model of collisionless plasma with finite Larmor radius effects, Phys. Plasmas, 2, 4451 .

Snyder, P. B., G. W. Hammett, and W. Dorland (1997), Landau fluid models of collisionless magnetohydrodynamics, Phys. Plasmas, 4, 3974 Southwood, D. J., and M. G. Kivelson (1993), Mirror Instability: 1. Physical mechanism of linear instability, J. Geophys. Res., 98(A6), 9181.

Stasiewicz, K. (2004), Reinterpretation of mirror modes as trains of slow magnetosonic solitons, Geophys. Res. Lett., 31, L21804, doi:10.1029/ 2004GL021282.

Stasiewicz, K. (2005), Ion-pressure equations derived from measurements in space, Phys. Rev. Lett., 95, 015, 004.

Treumann, R. A., C. H. Jaroschek, O. D. Constantinescu, R. Nakamura, O. A. Pokhotelov, and E. Georgescu (2004), The strange physics of lowfrequency mirror mode turbulence in the high temperature plasma of the magnetosphere, Nonlin. Processes Geophys., 11, 647, SRef-ID: $1607-$ 7946/npg/2004-11-647.

Tsurutani, B. T., E. J. Smith, R. R. Anderson, K. W. Ogilvie, J. D. Scudder, D. N. Baker, and S. J. Bame (1982), Lion roars and nonoscillatory drift mirror waves in the magnetosheath, J. Geophys. Res., 87(A8), 6060

Tsurutani, B. T., G. S. Lakhina, J. S. Pickett, F. L. Guarnieri, N. Lin, and B. E. Goldstein (2005), Nonlinear Alfvén waves, discontinuities, proton perpendicular acceleration, and magnetic holes/decreases in interplanetary space and the magnetosphere: Intermediate shocks?, Nonlin. Processes Geophys., 12, 321, SRef-ID: 1607-7946/npg/2005-12-321.

T. Passot and P. L. Sulem, CNRS, Observatoire de la Côte d'Azur, B.P 4229, 06304 Nice Cedex 4, France. (passot@obs-nice.fr; sulem@obs-nice.fr) 